UCRL-ID-129965

\title{
Preprocessing of Backprojection Images in the McClellan Nuclear Radiation Center Tomography System
}

\author{
Matthew R. Gibbons \\ Lawrence Livermore National Laboratory \\ P. O.Box 808, L-395 \\ Livermore, CA 9455I \\ Kevin Shields \\ McClellan Nuclear Radiation Center \\ SM-ALC/TIR, 5335 Price Ave. \\ McClellan AFB, CA 95652
}

February 19, 1998

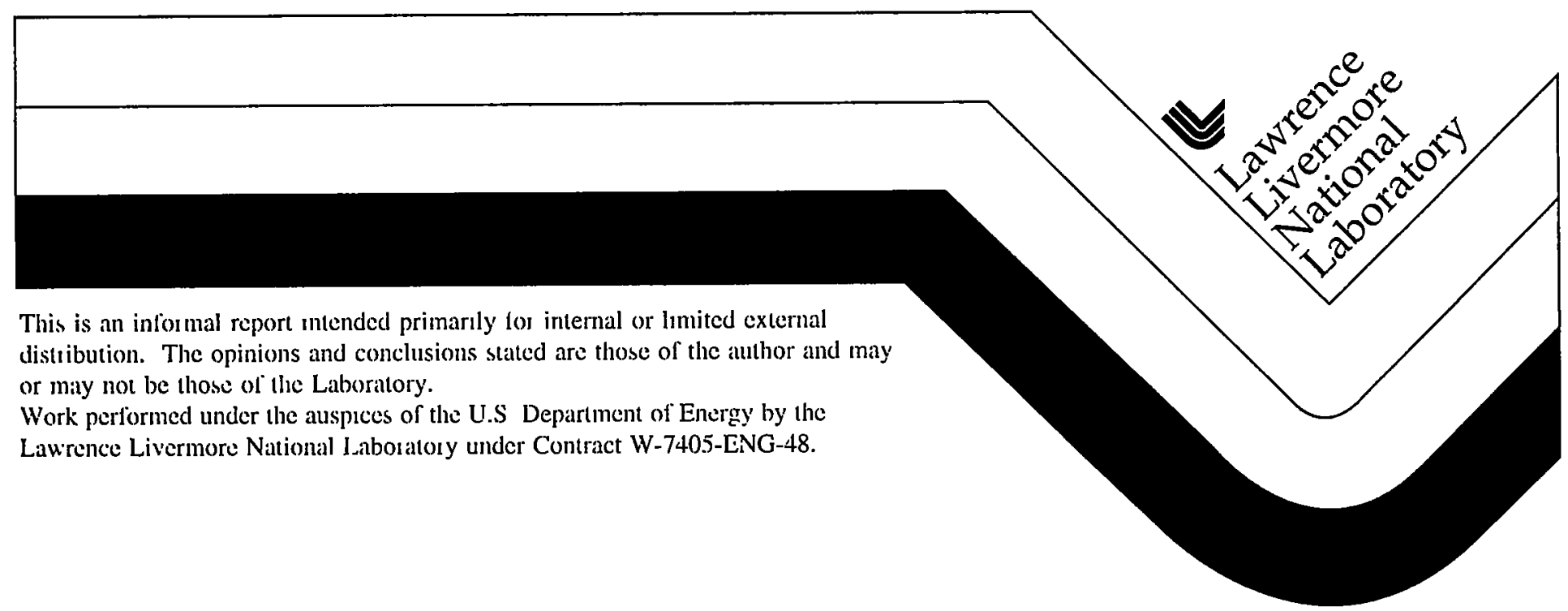




\section{DISCLAIMER}

This document was prepared as an account of work sponsored by an agency of the United States Government. Neither the United States Government nor the University of California nor any of their employees, makes any warranty, express or implied, or assumes any legal liability or responsibulity for the accuracy, completeness, or usefulness of any information, apparatus, product, or process disclosed, or represents that its use would not infringe privately owned rights. Reference herein to any specific commercial product, process, or service by trade name, trademark, manufacturer, or otherwise, does not necessarily constitute or imply its endorsement, recommendation, or favoring by the United States Government or the University of California. The views and opinions of authors expressed herein do not necessarily state or reflect those of the United States Government or the University of California, and shall not be used for advertising or product endorsement purposes.

This report has been reproduced directly from the best available copy.

Available to DOE and DOE contractors from the Office of Scientific and Technical Information

P.O. Box 62, Oak Ridge, TN 37831

Prices available from (615) 576-8401, FTS 626-8401

Available to the public from the

National Technical Information Service

U.S. Department of Commerce

5285 Port Royal Rd.

Springficld, VA 22161 


\title{
Prcprocessing of Backprojection Images in the McClellan Nuclcar Radiation Conter Tomograplyy System
}

\author{
Matthew R. Gibbons \\ Lawrence Livermore National Laboratory \\ P.O. Box 808, L-395 \\ Livcrmore, CA 94550 \\ Kevin Shields \\ McClellan Nuclear Radiation Center \\ 5335 Price Ave., McClellan AFB, CA 95652
}




\begin{abstract}
Neutron tomography is being investigated as a nondestructive technique for quantitative assessment of low atomic mass impurity concentration in metals. Neutrons maximize the sensitivity given their higher cross sections for low $\mathrm{Z}$ isotopes while tomography provides the three dimensional density information. The specific application is the detection of Hydrogen down to $200 \mathrm{ppm}$ weight in aircraft engine compressor blades. A number of preprocessing corrections have been implemented for the backprojection images in order to achieve the detection requirements for a testing rate of threc blades per hour. Among the procedures are corrections for neutron scattering and beam hardening. With these procedures the artifacts in tomographic reconstructions are shown to be less than the signal for $100 \mathrm{ppm}$ hydrogen in titanium alloy samples.
\end{abstract}





\section{Contents}

1 Introduction 5

2 Image Characteristics 9

2.1 Spatial Resolution and Focusing . . . . . . . . . . . . 9

2.1.1 Camera Focusing . . . . . . . . . . . . . . 9

2.1.2 Spatial resolution in the beam image . . . . . . . . . 9

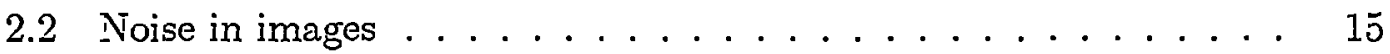

2.2 .1 Random errors . . . . . . . . . . . . 15

2.2 .2 Systematic errors . . . . . . . . . . . . 19

3 Preprocessing of images $\quad 23$

3.1 Correction of scattering and fluctuation errors . . . . . . . 23

3.2 Beam attenuation and hardening . . . . . . . . . . 25

3.3 The hydrogen signal ................. 30

4 Tomographic Reconstruction Results 33

4.1 NIST titanium alloy standard ............... 33

4.2 Cylindrical phantom . . . . . . . . . . . . . . 34

4.3 Compressor blade reconstruction ............ 39

5 Conclusion $\quad 41$

6 Acknowledgments $\quad 42$ 


\section{LIST OF FIGURES}

1.1 The MINRC neution tomography system $\ldots \ldots \ldots \ldots \ldots$

2.1 Focusing image generated with the $50 \mathrm{~mm}$ lens. . . . . . . . 10

2.2 Normalized beam intensity at the edge of a Cd square a) data, b) fit assuming beam divergence edge spread, and c) fit assuming general edge spread. . . . . . . . . . . . . . . . . 15

2.3 Image of a ruler behind the scintillation screen. . . . . . . . 20

3.1 Typical image of a compressur blade before noise correction and normalization. The drop in beam intensity can be seen at the edges of the image. The borated polyethylene can be seen as the dark stripe across the image. The rod to the left of the blade is a centering rod. The rod is removed during backprojection image acquisition. . . . . . . . . . . . . . 25

3.2 Neutron flux $\left(\frac{\#}{\pi} / \mathrm{cm}^{2} / \mathrm{MeV} / \mathrm{s}\right)$ energy spectrum in the neutron beam. 26

3.3 Attenuation coefficient $\left(\mathrm{cm}^{-1}\right)$ assuming exponential attenuation for a) Ti6Al4V and b) polyethylene. . . . . . . . . . . . 28

3.4 Dependence of intensity on Ti6Al4V thickness. The solid curve is the fit to the data points represented by squares. The dashed curve is the result assuming exponential attenuation and a constant attenuation coefficient. . . . . . . . . . . . 29

4.1 Reconstruction of the NIST Ti6 Al4V standard with a) no corrections and b) all corrections . . . . . . . . . . . . . 34

4.2 Attenuation coefficient across the center of the disk for the a) uncorrected and b) corrected backprojection images. . . . . . . .

4.3 Reconstruction of aluminum cylinder with Ti90Al6V4, $\mathrm{Ti}, \mathrm{Cu}, \mathrm{W}$, and $\mathrm{Ni}$ rods. A backprojection image is also shown. . . . . . . . 
4.4 The measured attenuation coefficient, normalized to the titanium alloy during image preprocessing, plotted versus the thermal neutron attenuation coefficient for several metals. . . . . . . . . 38

4.5 Tomographic reconstruction of a compressor blade. . . . . . . . 39

4.6 Cross section of engine compressor blade rcconstruction a) across the thickness of the blade and b) across the width of the blade. . 40 


\section{LIST OF TABLES}

2.1 Tomography system lens configurations . . . . . . . . . . . 11

2.2 Tomography system resolution . . . . . . . . . . . . . . 14

2.3 CCD camera dark charge . . . . . . . . . . . . . 16

2.4 CCD camera dark charge with bay shutter open . . . . . . . . 17

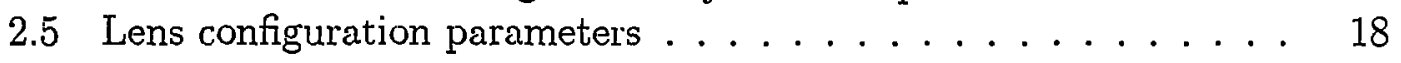

2.6 Beam random image noise . . . . . . . . . . . . . . . 19

2.7 Total random image noise . . . . . . . . . . . . . . . . . . 19

2.8 Neutron backscatter intensity .............. 21

3.1 Hydrogen normalized density . . . . . . . . . . . . 32

4.1 Relative attenuation coefficients for different metals . . . . . . . 37 


\section{INTRODUCTION}

Neutron radiography has already proven itself as a reliable method for the detection of hydrogenous substances, such as moisture and corrosion, in aircraft components[fro90]. Internal properties of objects are distinguished by differences in the attenuation coefficients of different materials. The advantage with neutrons is that low atomic number nuclei such as hydrogen have higher interaction cross sections than the surrounding metal. At the McClellan Nuclear Radiation Center (MNRC) four radiography bays use both film and real-time imaging devices to inspect aircraft components[ric90][alv90]. Radiographic images can be displayed in real-time at an operator's console as robots move parts across the neutron beam. Trained radiographers are able to rapidly identify regions of corrosion. Traditional radiography has limits since it only provides information on the total attenuation integrated over the path of the radiation through the material. The actual distribution of hydrogen across that path is not known precisely.

As an extension to radiographic methods the MNRC is investigating the use of neutron tomography to obtain quantitative hydrogen concentration data for aircraft engine compressor blades. The hydrogen concentration can be used as an indicator for the loss of mechanical properties, embrittlement, which can occur in metals after exposure to hydrogen[pat74][nel83]. In this case the defect to be identified is low concentrations of hydrogen diffused into the metal not corrosion or cracks. The signals to be detected are too small to be recognized from a traditional radiograph by the human eye. The goal of the tomography system is to provide a nondestructive inspection technique giving accurate hydrogen concentration data from several thousand down to 200 parts per million ( $\mathrm{ppm}$ ) weight for a total engine fan blade at, a rate of three blades per hour. The blades are composed of Ti6Al4V alloy with a height of $30 \mathrm{~cm}$, an average width of $9 \mathrm{~cm}$, and an average thickness of $0.64 \mathrm{~cm}$. The blades twist from top to bottom and have a crescent cross section precluding the use of simple radiography which might be appropriatic for a flat plate of metal.

The requirements for a system, that can achieve the above goal, result in 
cortain characteristics. The order of magnitude variation in spatial dimensions of the blades coupled with the rapid production rate favor a large neutron beam with a relatively high flux. The system must also be able to perform with a minimum number of tomographic projections to save time while maintaining a spatial resolution sufficient to resolve less than $0.5 \mathrm{~cm}$. The requirement to detect. the distribution of low hydrogen concentrations resulted, as mentioned previuusly, in the choice of tomography over radiography. In addition, errors and artifacts in the projections and reconstructions must be minimized to increase the signal to noise ratio for the calculated attenuation coefficients.

This paper describes the techniques used to preprocess the neutron radiographs so the resulting tomographic reconstructions meet the requirements for compressor blade evaluation. Preprocessing removes spurious signals caused by CCD camera dark charge, by beam intensity fluctuations, by background noise, by object neutron scattering, and by beam hardening. Reconstructions of various samples are shown to validate the techniques. The resulting neutron tomography system is applicable to a wide range of NDE tasks where rapid evaluation of low $\mathrm{Z}$ impurity concentrations is needed.

The tomography system consists of a neutron source, an object turntable, a scintillator screen, a mirror, a CCD camera, and computer support as shown in figure 1.1. The neutron source is a $2 \mathrm{MW}$ TRIGA thermal reactor. The neutron beam in radiography bay 3 is approximately $50 \mathrm{~cm}$ in diameter at the scintillation screen, and the ratio of the collimator aperture to screen distance over the collimator diameter is $235(653 \mathrm{~cm} / 2.8 \mathrm{~cm})$. The thermal neutron flux at $2 \mathrm{MW}$ reactor power is $1.2 \cdot 10^{7} \mathrm{n} / \mathrm{cm}^{2} \mathrm{~s}$ which is about $50 \%$ of the total flux in the beam. Four object turntables are fixed to a robot which can place them at different positions and orientations. Each turntable has fixtures that can accomodate up to three compressor blades. Normally one turntable is centered approximately 10 $\mathrm{cm}$ in front of the scintillator screen. A shutter blocks the beam port, when it is not in use, allowing access to the bay while the reactor is at power. With this bay configuration a dozen blades can be inspected, and then a new lot can be set in place without interrupting reactor operation.

The neutrons are detected with a scintillator screen which uses $\mathrm{Li}^{6}$ as the neutron absorber. Given the decrease in the $\mathrm{Li}^{6}$ absorption cross section with increasing energy, most of the neutrons detected are in the thermal region of the energy spectrum. The screen light output is $1.7 \cdot 10^{5}$ photons per neutron with an absolute scintillation efficiency of $9 \%$ and resolution limits of less than 100 $\mu \mathrm{m}[\mathrm{spo69}][$ net94]. 


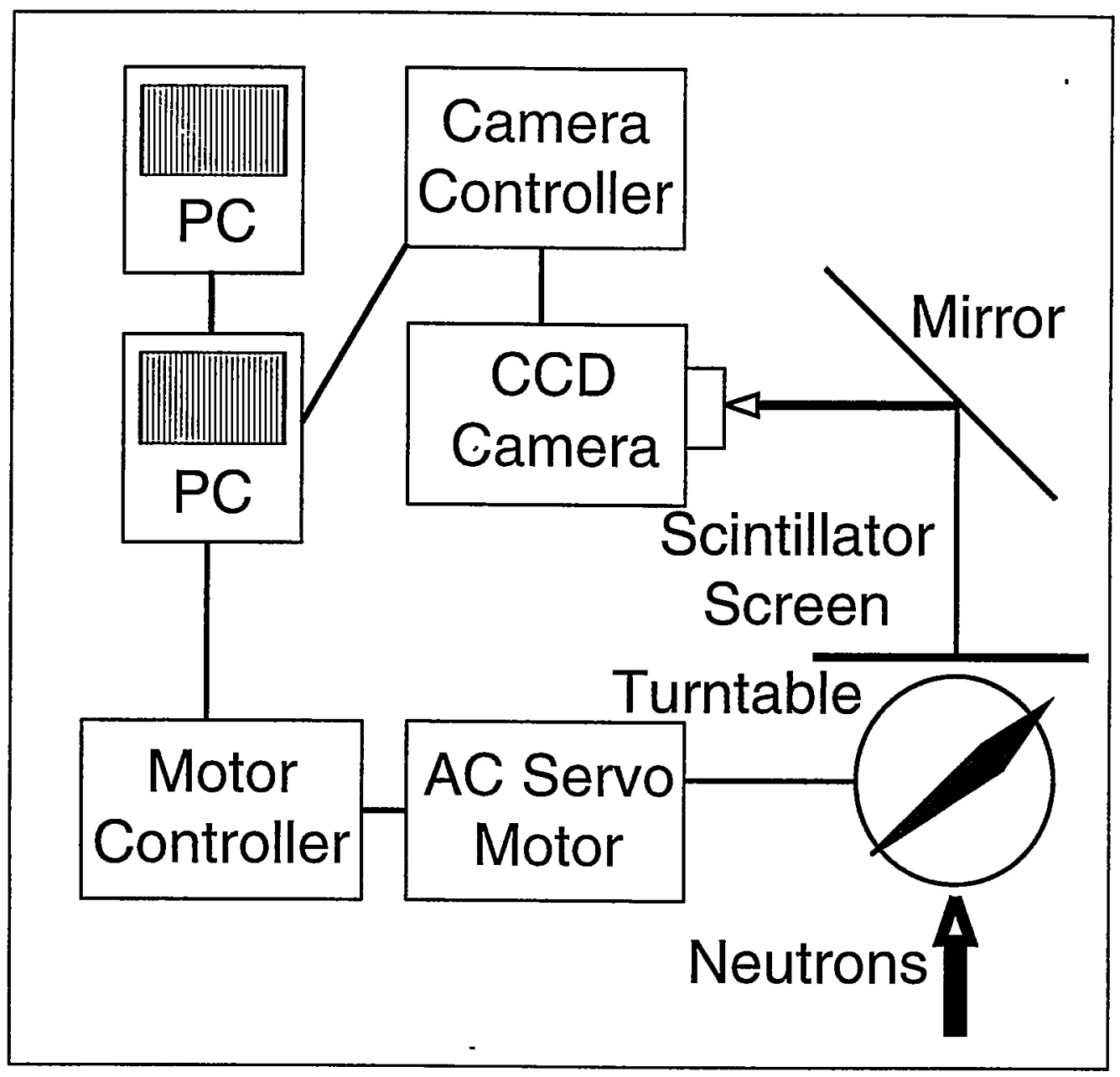

Figure 1.1: The MNRC neutron tomography system 
Photons generated in the screen are reflected from the mirror, pass through a lens and enter the CCD detector. Usually a $50 \mathrm{~mm}$ lens is used giving an image pixel size of $0.057 \mathrm{~cm}$. The CCD detector has a rectangular array of $1024 \times 1024$ pixels (24 $\mu \mathrm{m} \times 24 \mu \mathrm{m}$ pixels). The photoelectrons generated at each CCD pixel comprise the electrical signal and are proportional to the number of neutrons detected. The CCD quantum efficiency is $80 \%$ in the 0.6-0.7 $\mu \mathrm{m}$ range where the scintillator emits its light:[pim93]. The image data is collected in a 16 bit format.

Image acquisition and reconstruction is controlled with two workstations. One provides control to the CCD camera and sample turntable as well as image data capture and image analysis functions while the other provides tomographic reconstruction and image analysis functions. 


\section{Image Characteristics}

The characteristics of the radiographic imagess, nsed as backprojections for the tomographic reconstruction, set the fundamental limits on the accuracy of the reconstruction. The noise and resolution characteristics of the images are described in this chapter.

\subsection{Spatial Resolution and Focusing}

The size of detectable defects in tomographic reconstructions is limited by the spatial resolution of the initial backprojection images. Focusing procedures and edge spread in the images are summarized in this section. We have not found it necessary to make corrections of the resolution in the backprojection images. This section provides the information necessary to perform such corrections should they become needed.

\subsubsection{Camera Focusing}

The camera is focused by imaging a sheet of paper with a set of five converging lines. The paper is placed on the back of the scintillation screen, and the camera lens is focused so that the lines can be distinguished as close as possible to the convergence point. The pixel size is found by counting the number of pixels required to extend a length of 10 inches as measured by an imaged ruler. The pixel size and lens configurations are listed in table 2.1. A focusing image with the $50 \mathrm{~mm}$ lens is shown in figure 2.1 .

\subsubsection{Spatial resolution in the beam image}

After focusing is complete, the sharpness of an edge can be determined using neutron absorbing objects at various distances in front of the scintillation screen. Cadmium squares $4 \mathrm{~cm}$ across and $0.076 \mathrm{~cm}$ thick were placed at several distances in front of the screen. The intensity behind the $\mathrm{Cd}$ is approximately $10 \%$ of the 


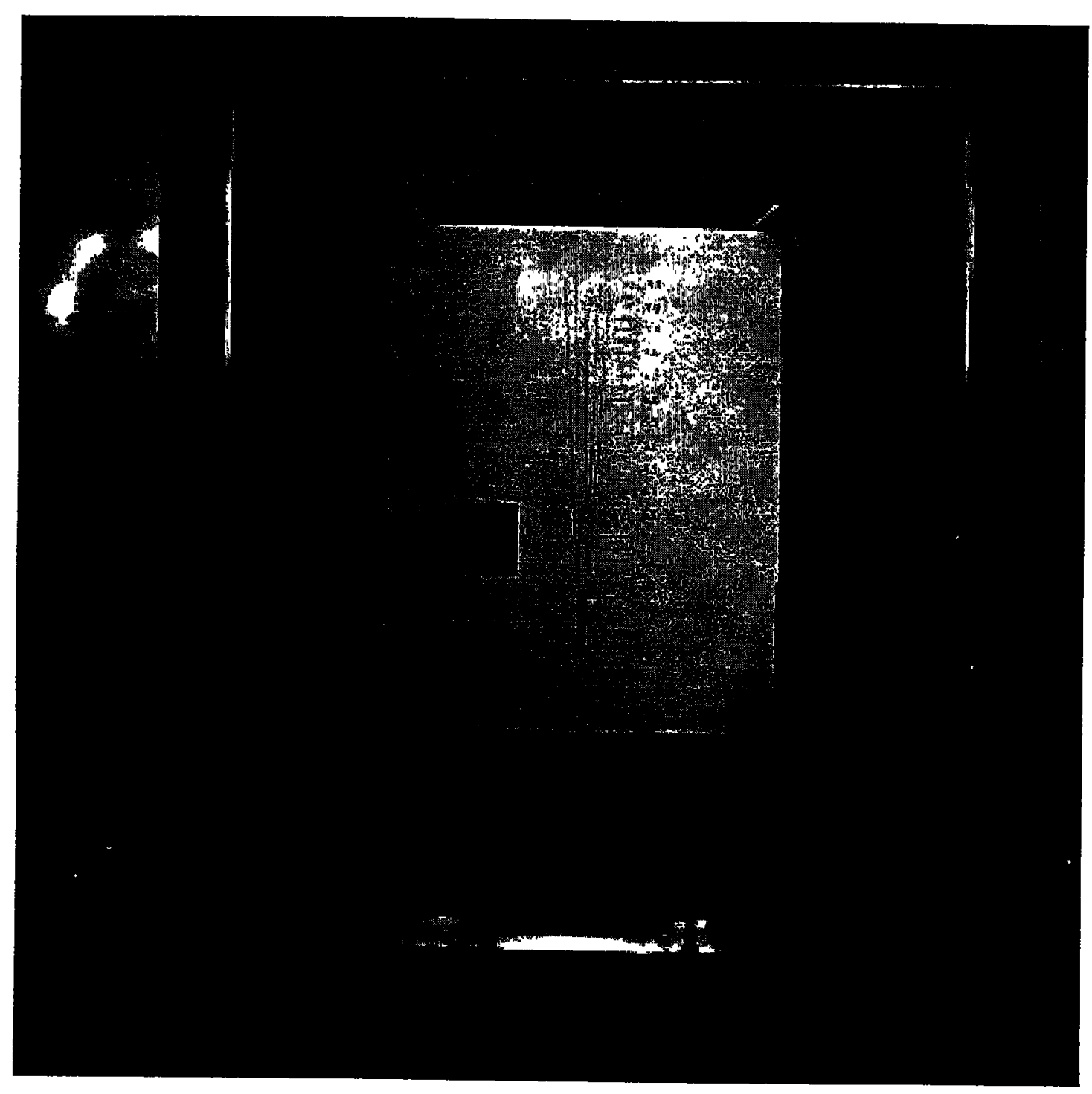

Figure 2.1: Focusing image generated with the $50 \mathrm{~mm}$ lens. 
Table 2.1: Tomography system lens configurations

$\begin{array}{ccc}\text { Lens } & \text { Configuration } & \text { Pixel size } \\ 50 \mathrm{~mm} & \text { I } & 0.0571 \mathrm{~cm} \\ 50 \mathrm{~mm} \text { and } 2 \mathrm{X} \text { teleconverter } & \text { II } & 0.0273 \mathrm{~cm} \\ 135 \mathrm{~mm} \text { and } 2 \mathrm{X} \text { teleconvarter } & \text { III } & 0.0102 \mathrm{~cm}\end{array}$

unimpeded beam intensity. The resolution is calculated by fitting an edge spread function to an intensity curve which crosses each square. The intensities are normalized using an image of the unperturbed beam, a flat field image.

The edge spread function is found from the point spread function, $P(x, y)$, assumed for the beam. As in Harms and Wyman[har86], the general point spread function, which may be due to converter unsharpness, scattering, diverging beam,etc., is

$$
P(x, y)=\frac{\lambda^{2}}{2 \pi\left[1+\lambda^{2}\left((x-\eta)^{2}+(y-\nu)^{2}\right)\right]^{3 / 2}}
$$

Here $\lambda$ is the resolution parameter, $x, y$ are the coordinates in the image plane, and $\eta, \nu$ are the coordinates in the object plane.

The flux distribution at the screen, $\Phi(x, y)$, is found from the convolution integral of the flux at the object, $\Psi$, and the point spread function. The integral has the form

$$
\Phi(x, y)=\frac{\lambda^{2}}{2 \pi} \iint \frac{\Psi(x-u, y-v) d u d v}{\left(1+\lambda^{2}\left(u^{2}+v^{2}\right)\right)^{3 / 2}}
$$

where $u=x-\eta$ and $v=y-\nu$. Assuming a constant flux, $I_{o}$, and integrating over du, we find

$$
\Phi(x, y)=\frac{\lambda^{2}}{2 \pi} \int \frac{I_{o} u d v}{\left(1+\lambda^{2} v^{2}\right)\left(1+\lambda^{2}\left(u^{2}+v^{2}\right)\right)^{1 / 2}}
$$

The final result is

$$
\Phi(x, y)=\frac{I_{o}}{2 \pi} \arctan \left(\frac{\lambda^{2} u v}{\left(1+\lambda^{2}\left(u^{2}+v^{2}\right)\right)^{1 / 2}}\right)
$$

Consider a rectangle of material which attenuates the normalized signal to the level $\alpha$ and has spatial extent from $x_{m}$ to $x_{p}$ and $y_{m}$ to $y_{p}$. The function of eq.(2.4) 
must be integrated over five regions. The result along $y=0$ for a rectangle of material extending from $-b$ to $b$ in $y$ is

$$
\Phi(x, 0)=1+\frac{1}{\pi}(1-\alpha)\left[\begin{array}{c}
\arctan \left(\frac{\lambda^{2}\left(x-x_{\eta}\right) b}{\left(1+\lambda^{2}\left(\left(x-x_{p}\right)^{2}+b^{2}\right)\right)^{1 / 2}}\right) \\
-\arctan \left(\frac{\lambda^{2}\left(x-x_{m}\right) b}{\left(1+-\lambda^{2}\left(\left(x-x_{m}\right)^{2}+b^{2}\right)\right)^{1 / 2}}\right)
\end{array}\right]
$$

The spatial resolution, defined as the distance over which the intensity decreases from $.75(1-\alpha)+\alpha$ to $.25(1-\alpha)+\alpha$. is approximately $2 / \lambda$. Note that the rosult in the limit of $b \rightarrow \infty$ is

$$
\Phi(x, 0)=1+\frac{1}{\pi}(1-\alpha)\left[\arctan \left(\lambda\left(x-x_{p}\right)\right)-\arctan \left(\lambda\left(x-x_{m}\right)\right)\right]
$$

A Levenberg-Marquardt[pre88] algorithm was used to find a best $\chi^{2}$ fit to the data with $\alpha, \lambda, x_{m}, x_{p}$ as parameters for the nonlinear edge spread equation. The limiting equation, (2.6), was found to be sufficient since the vertical extent of the object would have to be less than $0.5 \mathrm{~cm}$ before the results are significantly affected.

A significant portion of the edge spread is due to the divergence of the neutron beam. The flux profile due to diverging beam edge spread is flatter behind the absorbing object than that resulting from the general edge spread function. Since this project requires the accurate determination of small changes in attenuation, it is important to consider the edge spread function specific to a diverging beam. For this work consider a strip beam of half width $a$.

$$
\Phi(x)=\frac{\lambda I_{o}}{\pi} \int_{-a}^{a} \frac{\Psi(x+\eta l / L) d \eta}{1+\lambda^{2}(x-\eta)^{2}}
$$

Here $L$ is the beam collimator plane to object plane separation, $l$ is the object plane to image plane separation, and $\lambda=1 /(L+l)$.

If there is no object in the beam the flux is

$$
\Phi(x)=-\frac{I_{0}}{\pi}\left[\arctan \left(\frac{x-a}{L+l}\right)-\arctan \left(\frac{x+a}{L+l}\right)\right]
$$

At $x=0$ this becomes

$$
\Phi(0)=\frac{2 I_{o}}{\pi} \arctan \left(\frac{a}{I+l}\right)
$$


Now consider an absorbing object, with attenuation $\alpha$ positioned from $x=-\infty$ to $x=0$. The image flux becomes

$$
\Phi(x)=\frac{\lambda I_{o}}{\pi} \int_{-a}^{a} \frac{1}{1+\lambda^{2}(x-\eta)^{2}}[\alpha I I(-x-\eta l / L)+x+\eta l / L] d \eta
$$

where $\mathrm{H}(\mathrm{x})$ is the Heaviside function. The solution is

$$
\Phi(x)=\left\{\begin{array}{cr}
-\frac{\alpha}{\pi}[\arctan (\lambda(x-a))-\arctan (\lambda(x+a))] & x L / l \leq-a \\
-\frac{1}{\pi}[\arctan (\lambda(x-a))-\arctan (\lambda x(1+L / l))] \\
-\frac{\alpha}{\pi}[\arctan (\lambda x(1+L / l))-\arctan (\lambda(x+a))] \\
-\frac{1}{\pi}[\arctan (\lambda(x-a))-\arctan (\lambda(x+a))] & x L / l>-a \\
& x L / l>a
\end{array}\right.
$$

The total width of the intensity transition region from $\alpha$ to 1.0 is approximately $2 a l / I$ for divergences small compared to $a$. The spatial resolution is given by $a l / L$. For the MNRC reactor $a=1.41 \mathrm{~cm}$ (before the 1996 upgrade to $2 \mathrm{MLW}$ $a=2.81 \mathrm{~cm}$ ) and $L=653 . \mathrm{cm}$. This function, unlike the general point spread function, results in a penumbra which does not extend beyond the spatial resolution distance.

The edge spread results for several Cd positions are shown in table 2.2. Column three of the table lists the results from fits of the general edge spread function to the experimental data, and column four lists the expected result for beam divergence. The resolution is worse than what is predicted from beam divergence alone. Results before and after the reactor upgrade from IMW to $2 \mathrm{MTW}$ are listed. With the upgrade the collimator diameter was decreased to maintain the same flux at the end of the beam tube. The resolution has become finer but not by a factor of two. This result is consistent with edge spreads haring sources other than beam divergence. The important result for the tomography project is that objects centered at $10 \mathrm{~cm}$ from the screen have resolutions of approximately $0.5 \mathrm{~mm}$.

A comparison between the data and the fit for the $10.0 \mathrm{~cm}$ position, $2 \mathrm{MW}$ case is shown in figure 2.2. The data intensity is essentially flat behind the cadmium. This behaviour is best fit by the edge spread function specific to beam divergence; however, the collimator diameter used to achieve this fit was significantly larger $(13.4 \mathrm{~cm})$ than the actual collimator. The edge spread appears to have contributions from other sources which worsen the resolution, but keep the intensity behind the object relatively flat. The general edge spread function tends to cause a small dip in intensity of about $1 \%$ behind the object. This could 
Table 2.2: Tomography system resolution

$\begin{array}{cccc}\begin{array}{c}\text { Cd Position } \\ (\mathrm{cm})\end{array} & \begin{array}{c}\lambda(\mathrm{cm}-1) \\ \text { general }\end{array} & \begin{array}{c}\text { resolution }(\mathrm{cm}) \\ \text { general }\end{array} & \begin{array}{c}\text { resolution }(\mathrm{c}) \\ \text { geometric }\end{array} \\ & & & \\ 1 \mathrm{MIW} & & & \\ \text { on screen } & 44.83 & 0.0446 & 0.0 \\ 4.0 & 30.17 & 0.0663 & 0.0172 \\ 11.5 & 27.97 & 0.0715 & 0.0495 \\ 31.5 & 20.45 & 0.0978 & 0.136 \\ 2 \mathrm{MWW} & & & \\ \text { on screen } & 49.63 & 0.0403 & 0.0 \\ 10.0 & 33.11 & 0.0604 & 0.0215 \\ 30.0 & 24.21 & 0.0826 & 0.0645\end{array}$

be a matter of concern since the signals to be detected with the system require the intensity to be accurate to less than $1 \%$. As shall be shown in chapter 3 , the intensity information in images will be related to object thickness through a table of attenuation vs thickness. Images which have been corrected for edge spread must use material thickness data from a table generated using calibration images which were corrected for edge spread in the same manner.

Correction of images can be accomplished by considering the convolution integral resulting in the image flux

$$
\Phi(x, y)=\Psi(x, y) * P(x, y) .
$$

The Fourier transform of the convolution is

$$
\operatorname{FT}\left[\Phi\left(k_{x}, k_{y}\right)\right]=\operatorname{FT}\left[\Psi\left(k_{x}, k_{y}\right)\right] * \operatorname{FT}\left[P\left(k_{x}, k_{y}\right)\right] .
$$

So the actual object intensity distribution is found from the inverse Fourier transform, IFT,

$$
\Psi(x, y)=I I T\left[F T\left[\Phi\left(k_{x}, k_{y}\right)\right] / F T\left[P\left(k_{x}, k_{y}\right)\right]\right] .
$$

The Fourier transform of the point spread function is called the modulation transfer function, MTF. A filter function may be necessary to limit anomolously large signals at high frequency due to noise in the image signal. The only quantity needed for these corrections is the resolution parameter given in table 2.2. Since the resolution is about the same as the pixel size, edge spread corrections have not been incorporated into the image preprocessing at the present time. 


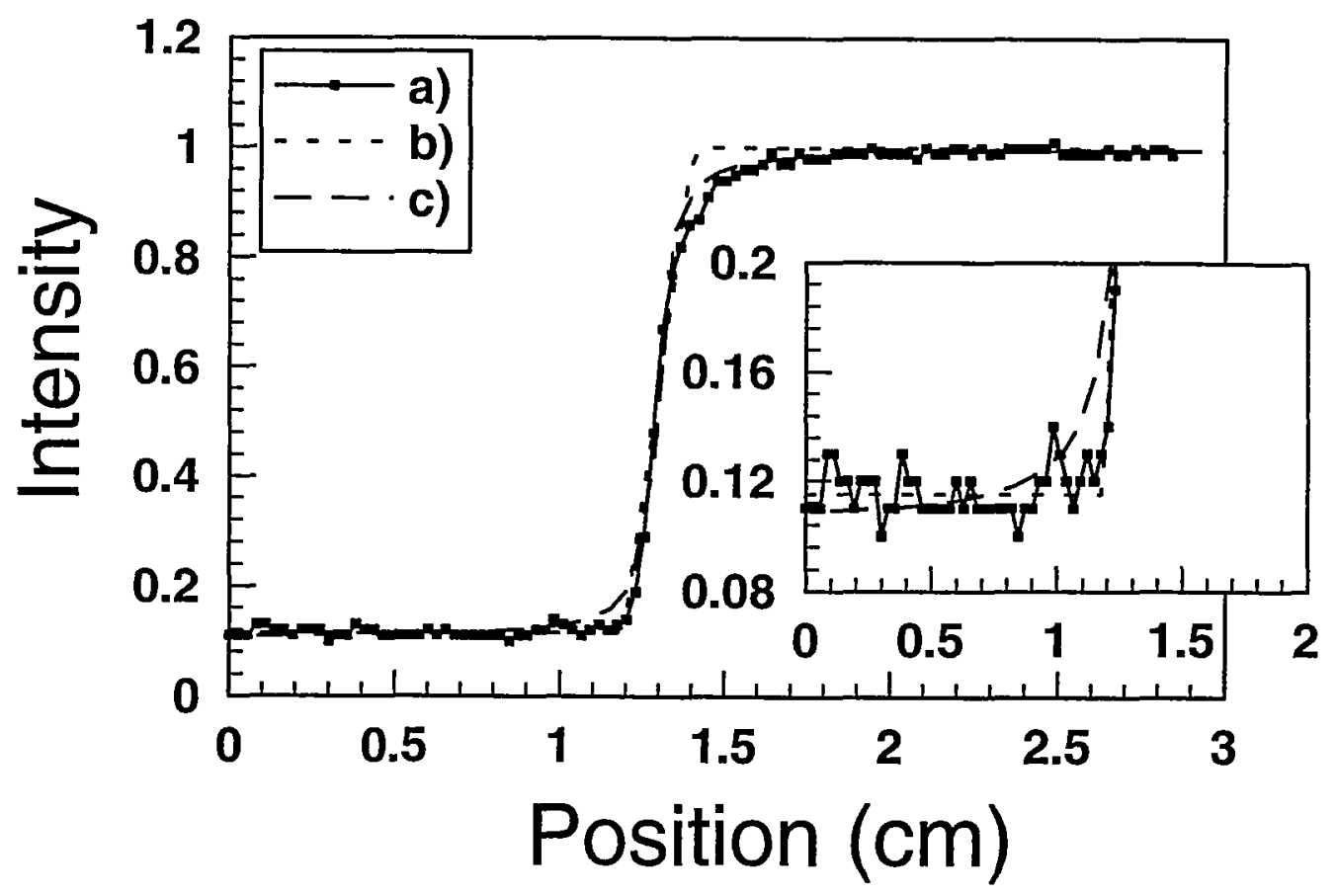

Figure 2.2: Normalized beam intensity at the edge of a Cd square a) data, b) fit assuming beam divergence edge spread, and c) fit assuming general edge spread.

\subsection{Noise in images}

Noise will exist in any image taken using the system. This section identifies the sources of noise, its magnitude, and pussible improvements. We shall note the contribution of the noise sources to either random or systematic errors. Random error measurements will be used to determine the limit on the minimum signal magnitude while systematic error measurements will provide the parameters necessary for image correction.

\subsubsection{Random errors}

The pixels in a CCD experience a thermally induced buildup of charge as a function of time. Each pixel will tend to build up charge at a slightly different rate as 
Table 2.3: CCD camera dark charge

$\begin{array}{ccc}\text { Exposure (sec) } & \begin{array}{c}\text { Intensity } \\ \text { mean }\end{array} & \begin{array}{c}\text { Intensity } \\ \text { standard deviation }\end{array} \\ & & \\ 5 & 247 . & 48.2 \\ 10 & 339 . & 86.4 \\ 20 & 527 . & 175 . \\ 40 & 935 . & 365 .\end{array}$

well as suffer from statistical noise within each pixel. The manufacturer quotes a rate of 3-6 electrons/pixel/sec when this camera is operated at $-50{ }^{\circ} \mathrm{C}$ which corresponds to 0.5 counts $/ \mathrm{pixel} / \mathrm{sec}[\mathrm{pim} 93]$. This gives 6 to 12 electrons for each intensity value. Thus a signal of 50,000 represents 300,000 to 600,000 electrons. There is an increase of the dark charge rate by a factor of approximately two for every $7{ }^{\circ} \mathrm{C}[\mathrm{pim} 93]$. Our operating temperature of $-27^{\circ} \mathrm{C}$ should give a dark charge signal of approximately 5 counts $/ \mathrm{pixel} / \mathrm{sec}$.

Assuming a Gaussian distribution for the variation of dark charge between the pixels with mean, $\mu$, and standard deviation, $\sigma$, the distribution will remain the same as long as the ratio, $\mu / \sigma$, is constant for various sample times. In addition a dark charge rate of electrons/pixel/sec implies $\mu$ has a linear dependence on time. The dark charge was measured by acquiring images over several time intervals with the camera shutter closed and the radiation bay shutter closed. The measured intensities are listed in table 2.3. A linear fit to the data gives the magnitude of the dark charge as a function of time, $l$, in seconds

$$
\begin{aligned}
& I_{\mu}=19.71 \iota+142 . \\
& I_{\sigma}=9.12 \iota-2.38
\end{aligned}
$$

The offset from zero is probably due to electronic noise in the system. The rate of 20 counts/pixel/sec is a factor of four larger than the manufacturer estimate.

With the bay shutter open, scattered radiation from the neutron beam can interact with the CCD camera even with the camera shutter closed. This will add another noise component to the images. Table 2.4 lists the image characteristics with the camera shutter closed and the bay shutter open. A linear fit to this data results in

$$
I_{\mu}=25.09 t+183 .
$$


Table 2.4: CCD camera dark charge with bay shutter open

$\begin{array}{ccc}\text { Exposure (sec) } & \begin{array}{c}\text { Intensity } \\ \text { mean }\end{array} & \begin{array}{c}\text { Intensity } \\ \text { standard deviation }\end{array} \\ 5 & 310 . & 157 . \\ 10 & 433 . & 201 . \\ 20 & 684 . & 305 . \\ 40 & 1190 . & 506 . \\ & & I_{\sigma}=10.1 t+104 .\end{array}$

With the bay shutter open the rate of dark charge buildup is approximately the same, but the scattered radiation causes an additional signal offset of 40 . The rate of dark charge accumulation appears to be consistent so a dark charge image can be subtracted from neutron beam images to remove this unwanted signal. The uncertainty is quantified by the standard deviation, $\sigma$, in the dark charge signal. It must be remembered that the standard deviation is the variation in dark charge from pixel to pixel not the variation for different exposures in the same pixel. However, requiring signals to be larger than $\sigma$ for a given exposure time serves as a standard quaranteeing a signal larger than the noise. Thus eq. (2.16) indicates the magnitude required for the signal to be above the noise. As an example, a two byte signal should be accumulated in less than 22 seconds to keep the noise fraction under 0.005 .

It is possible that sources of light within the bay can cause a noise signal in images. A 10 second exposure was taken with the camera shutter open and the bay shutter closed. The average intensity and standard deviation were 435 . and 96.1 respectively. Since there is a negligible difference from the case with the camera shutter closed, the bay appears to be sufficiently light tight.

The neutron flux reaching the screen also suffers from statistical fluctuations. These fluctuations can be estimated from the number of electrons, $N_{e}$, produced in the CCD pixels by the beam. $N_{e}$ is given by[mof91]

$$
N_{c}=L \epsilon_{L} \epsilon_{Q}\left(N_{p} \epsilon_{s} \Phi \Lambda l\right)=\alpha\left(N_{p} N_{n}\right) .
$$

Here $L$ is the fraction of light captured by the lens system. $\epsilon_{L}$ is the lens transmission coefficient (0.85). $\epsilon_{Q}$ is the CCD quantum efficiency (0.8)[pim93]. 
Table 2.5: Lens configuration parameters

Configuration $f$ number minification $\quad$ L

$\begin{array}{cccc}\text { I } & 1.2 & 23.75 & 2.83 \cdot 10^{-4} \\ \text { II } & 2.1 & 11.38 & 3.71 \cdot 10^{-4} \\ \text { III } & 2.0 & 4.25 & 2.27 \cdot 10^{-3}\end{array}$

$N_{p}$ is the number of photons generated per neutron $\left(1.7 \cdot 10^{5}\right)\left[\right.$ spo69]. $c_{s}$ is the scintillation screen efficiency (0.09)[spo69]. $\Phi$ is the neutron flux $\left(1.2 \cdot 10^{7} \mathrm{n} / \mathrm{cm}^{2} \mathrm{~s}\right)$. $A$ is the pixel area $\left(0.0032 \mathrm{~cm}^{2}\right) . t$ is the exposure time.

For a Lambertian radiator[nus68]

$$
L=\frac{1}{(2 F(m+1))^{2}} .
$$

Here $F$ is the lens $f$ number, and $m$ is the minification or the ratio of the pixel size on the screen to the CCD pixel size $(0.0024 \mathrm{~cm})$. Table 2.5 lists the parameters for our lens system.

The standard deviation, $\sigma_{e}$, for the electron number is found from standard error analysis to be

$$
\sigma_{e}^{2}=\alpha^{2}\left[N_{n} \sigma_{p}^{2}+N_{p} \sigma_{n}^{2}\right] .
$$

Assuming a normal distribution for $N_{n}$ and $N_{p}$, the fractional standard deviation becomes

$$
\frac{\sigma_{e}}{N_{e}}=\left[\frac{1}{N_{p}}+\frac{1}{N_{n}}\right]^{1 / 2} .
$$

Table 2.6 has the image intensity and fractional standard deviation for the various lens configurations. The calculated intensity is found from the electron number by assuming 12 electrons per intensity value. The calculated intensities agree sufficiently with the measured intensities to give us confidence in the beam noise estimates. The calculated beam noise will be combined with the calculated dark charge noise to estimate the total random noise in image intensities.

The random errors of the dark charge and high frequency beam noise can be added in quadrature and compared to measured values. The dark charge noise is calculated using eq. (2.16) and the exposure times required to get the 55000 average beam intensity. The beam noise is taken from table 2.6. The measured 
Table 2.6: Beam random image noise

$\begin{array}{cccccccc}\text { Configuration } & \alpha & N_{n} & N_{e} & \begin{array}{c}\text { Calculated } \\ \text { Intensity }\end{array} & \begin{array}{c}\text { Vieasured } \\ \text { Intensity }\end{array} & \begin{array}{c}\text { Aquisition } \\ \text { Time (sec) }\end{array} & \sigma_{e} / N_{e} \\ & & & & & & & \\ \text { I } & 1.92 \cdot 10^{4} & 3.4 \cdot 10^{4} & 1.1 \cdot 10^{6} & 92000 . & 55000 . & 8.2 & 0.0060 \\ \text { II } & 1.26 \cdot 10^{-4} & 6.0 \cdot 10^{1} & 1.3 \cdot 10^{6} & 1.1 \cdot 10^{5} & 55000 . & 64 . & 0.00417\end{array}$

Table 2.7: Total random image noisc

$\begin{array}{cccc}\text { Configuration } & \begin{array}{c}\text { Exposure } \\ \text { Time (sec) }\end{array} & \begin{array}{c}\text { Random Error } \\ \text { (calculated) }\end{array} & \begin{array}{c}\text { Random Error } \\ \text { (measured) }\end{array} \\ \text { I } & 8.2 & 0.0069 & 0.0062 \\ \text { II } & 64 . & 0.014 & 0.015\end{array}$

noise values are found by dividing two images taken with the same exposure time and normalized to an average intensity of one. The error, $\sigma_{e} / N_{e}$, is assumed to be the standard deviation of the pixel intensities from the average of one. The results are shown in table 2.7. The beam noise is not much different than the dark charge noise, and both are usually only a few tenths of one percent. This bodes well for small signal detection, but leaves no clear avenue for random noise reduction. Longer exposures will decrease the beam noise, but this is offset by an increase in the dark charge noise. The optimum choice for random noise reduction would be an increase in beam flux.

\subsubsection{Systematic errors}

Light caused by the beam on the scintillation screen may scatter off of surfaces and reach the camera. To quantify this noise a ruler was placed on the back of the scintillation screen and imaged for 6.5 seconds. The result for lens configuration I is shown in figure 2.3. After the dark charge was subtracted a signal of up to 800 exists behind this opaque object. With a maximum beam intensity of 50,000 this represents a noise signal on the order of $1.5 \%$ or $0.0022 l$ with $l$ in seconds. Reflection of light off of the screen frame and other surfaces may be the cause of this noise. 

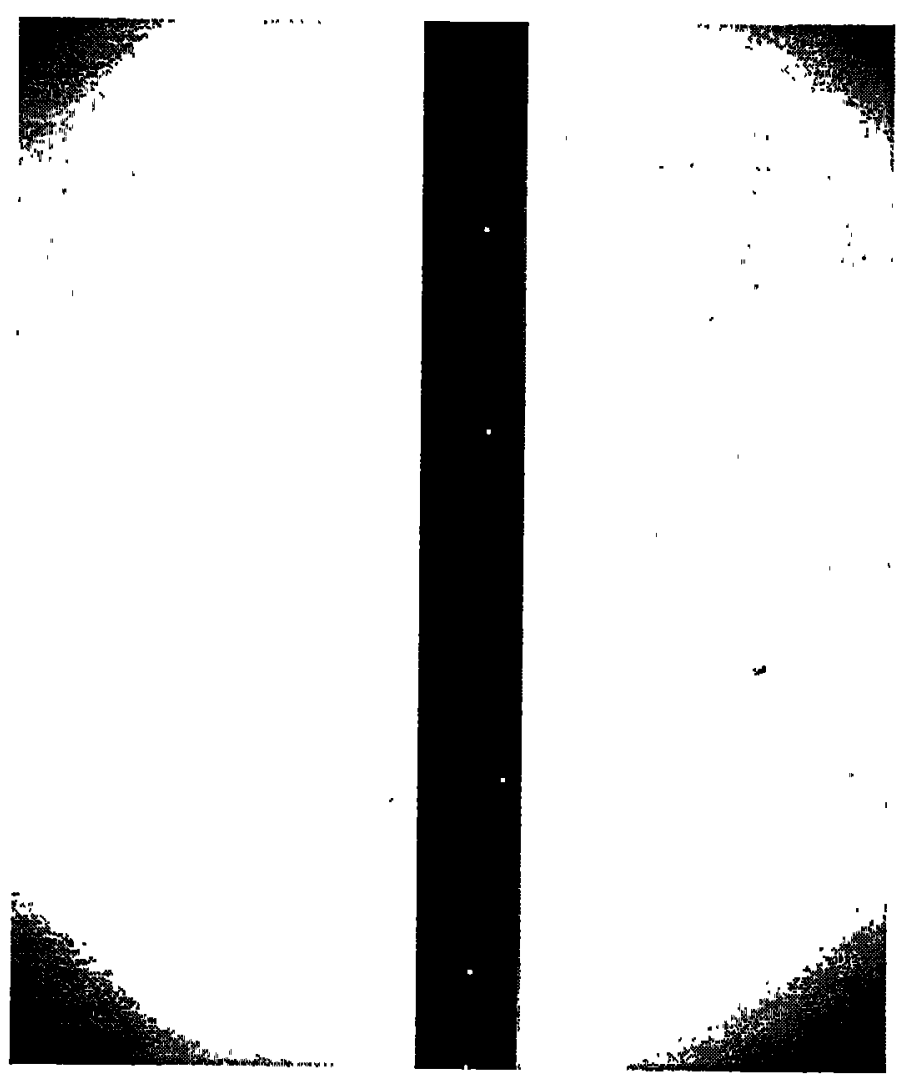

Figure 2.3: Image of a ruler behind the scintillation screen. 
Table 2.8: Neutron backscatter intensity

$\begin{array}{cccc}\begin{array}{c}\text { Lens } \\ \text { Configuration }\end{array} & \begin{array}{c}\text { Exposure } \\ \text { Time (sec) }\end{array} & \begin{array}{c}\text { Normalized } \\ \text { Intensity }\end{array} & \begin{array}{c}\text { Scattered Light } \\ \text { Intensity }\end{array} \\ \text { I } & 8.2 & 0.05347 & 0.018 \\ \text { II } & 64 . & 0.06273 & 0.026\end{array}$

As a further check an image using lens configuration II was taken with a 55 sccond exposure in order to obtain the 50,000 intensity level in each pixel. Again a substantial intensity exists behind the ruler (a fraction of 0.022 or $0.0004 t$ ).

Another noise signal can be found by placing a 1" thick strip of $30 \%$ borated polyethylene on the beam side of the scintillation screen. The borated polyethylene absorbs all of the neutrons incident on it. Any intensity measured behind the strip is noise. The normalized intensities behind the strip are shown in table 2.8. The last column in the table is the expected intensity due to the scattered light of the previous section. These background noise measurements are taken without an object in the beam. The borated polyethylene strip will also provide information on the object neutron scattering, but we separate the signals during preprocessing of images. The treatment of both signals will be discussed in the section on image preprocessing. This method is similar to Ikeda, et. al. [ike96]; however, they did not separate the background and object scatter signals.

The majority of the signal behind the borated polyethylene is not due to scattered light. The cause may be backscattered neutrons from the beam stop, mirror, and other structures which intersect the beam. The flux of scattered neutrons at the screen will result in an added signal from the screen independent of objects in the beam. Measurements of the neutron flux were taken outside of the beam path. These measurements were taken before and after installation of shielding for the CCD camera, and they are an indicator of the backscatter.

For a point $348 \mathrm{~cm}$ from the beam exit and $48 \mathrm{~cm}$ from the beam center, the flux was $3.3 \cdot 10^{6} \mathrm{n} / \mathrm{cm}^{2} \mathrm{~s}$. As a point of reference, the scintillation screen is $330 \mathrm{~cm}$ from the beam exit and at the beam center. The beam stop is 446 . $\mathrm{cm}$ from the beam exit or 116 . $\mathrm{cm}$ from the screen. The location of the screen versus the location of the flux measurement point will alter the flux at the screen compared to the flux measurement point. With this in mind the measurement will be used as an approximation of the backscatter flux at the screen. This 
measurement was taken outside of the camera shielding before a reentrant beam stop was installed. Inside the shield the flux was found to be $10^{5} \mathrm{n} / \mathrm{cm}^{2}$ s. After installation of the reentrant beam stop, the flux inside the shield became 2.5. $10^{4} \mathrm{n} / \mathrm{cm}^{2} \mathrm{~s}$. Assuming the same decrease in the flux outside of the shielding, the approximate backscatter flux at the screen becomes $8.3 \cdot 10^{5} \mathrm{n} / \mathrm{cm}^{2} \mathrm{~s}$. Comparing to the beam flux of $1.2 \cdot 10^{7} \mathrm{n} / \mathrm{cm}^{2} \mathrm{~s}$, the calculated fractional backscatter signal is 0.068 . This calculated signal is quite close to the part of the signal behind the borated polyethylene not taken into account by the scattered light signal. The problem with this approximaticly constant background is the net decrease in attenuation detected for an object placed in the neutron beam. This is equivalent to part of the beam never being attenuated by objects which could lead to beam hardening artifacts in reconstructed tomographic images[kak88]. The large size of the neutron backscatter and scattered light signals is a result of the large area of the beam. A small area beam would have much smaller scatter noise due to the isotropic scattering of the neutrons and light. The advantage, that size gives for fast processing of large objects, adds an extra noise signal which must be removed.

The analysis of the random error section assumed a constant flux for the beam on the time scale of image aquisition. It also indicates the fluctuations between pixels for images exposed to the same fluence eventhough the fluxes and aquisition times might be different. In the actual system the aquisition times are constant, but the average flux from the MNRC reactor oscillates over time. This is indicated by rariations of up to $\pm 5 \%$ in the average intensity of flat field images. These variations are much larger than the fluctuations indicated in the previous section. The variation manifests itself as an arerage variation of the pixel intensities between images not as a larger fluctuation between pixels in the same image. These variations must be taken into account when images are normalized by the flat field. 


\section{Preprocessing of images}

\subsection{Correction of scattering and fluctuation errors}

As a first step toward tomographic reconstruction, an accurate measure of the beam attenuation caused by an object is required. The objectire is to find the intensity behind the object, $I_{o}$, normalized by the beam intensity, $I_{b}$, despite corruptions due to system noise, image spreading, object scattering, and beam energy spread. An initial estimate can be found by only considering the dark charge intensity, $I_{d}$, the background noise, $I_{n}$, the object scattered beam intensity, $I_{s}$, and the fluctuation of the beam magnitude, $f$. The dark charge is subtracted during image acquisition. The portion of the dark charge signal aflected by beam fluctuations is small so those fluctuations are neglected. We shall consider the rest of the quantities to be spatially independent.

The intensity actually measured in the flat field image, $I_{b f}$, becomes

$$
I_{b f}=I_{b}+I_{n} .
$$

As mentioned above a borated polyethylene strip blocks part of the beam. This portion of the image acts as a scattering detector. Without an object in the beam there is no object scattering so the intensity behind the strip, $I_{s f}$, is just the background intensity

$$
I_{s f}=I_{n} .
$$

Next the intensities in the object image are considered. A typical image with the borated polyethylene strip and an engine compressor blade is shown in figure 3.1. The intensity in an unobstructed portion of an object image, $I_{b o}$, is

$$
I_{b o}=(1+f)\left(I_{b}+I_{n}+I_{s}\right) .
$$

The intensity behind the object in an object image, $I_{o o}$, is

$$
I_{o o}=(1+f)\left(I_{o}+I_{n}+I_{s}\right) \text {. }
$$


The scattering detector has an intensity in an object image, $I_{s n}$, of

$$
I_{s o}=(1+f)\left(I_{n}+I_{s}\right) .
$$

Now define $\delta_{b o f}$ as $I_{b o} / I_{b f}, \delta_{o o f}$ as $I_{o o} / I_{b f}$, and $\delta_{s o f}$ as $I_{s o} / I_{s f}$. These quantities are found from the quotient of the object image and the flat field image. Also define $\delta_{o}$ as $I_{o} / I_{b}, \delta_{n}$ as $I_{n} / I_{b}$, and $\delta_{s}$ as $I_{s} / I_{b}$. Note that $\delta_{n}$ was measured carlier in the section on background noise. Dividing the object image by the flat field image, equations (3.3), (3.4), and (3.5) become

$$
\begin{gathered}
\delta_{\text {bof }}=(1+f)\left(1+\delta_{n}+\delta_{s}\right) /\left(1+\delta_{n}\right) \\
\delta_{o o f}=(1+f)\left(\delta_{o}+\delta_{n}+\delta_{s}\right)
\end{gathered}
$$

and

$$
\delta_{s o f}=(1+f)\left(1+\delta_{s} / \delta_{n}\right)
$$

The drop in beam intensity at the edge of the image, as shown in figure 3.1, is the most compelling reason for normalization of the object images by the flat field image. We can solve for $\delta_{s}$ using eq. (3.6)

$$
\delta_{s}=\left(1+\delta_{n}\right) \delta_{b o f} /(1+f)-1-\delta_{n} .
$$

Placing this into eq. (3.8) results in a solution for $(1+f)$ in terms of $\delta_{b o f}$ and $\delta_{\text {sof }}$

$$
(1+f)=\left(1+\delta_{n}\right) \delta_{b o f}-\delta_{n} \delta_{\text {sof }}
$$

Given $(1+f)$ and $\delta_{s}$, the object intensity normalized by the beam intensity, $\delta_{o}$, becomes

$$
\delta_{o}=\left(1+\delta_{n}\right) \delta_{o o f} /(1+f)-\delta_{n}-\delta_{s} .
$$

The procedure for determining $\delta_{o}$ is

1) Divide the object image by the flat field image.

2) Find $\delta_{\text {bof }}$ by averaging the intensity for a region of pixcls that are not blocked by the scattering detector or by the object to be reconstructed.

3) Find $\delta_{\text {sof }}$ by averaging the intensity for a region of pixels that are located behind the scattering detector.

4) Calculate $(1+f)$ and $\delta_{s}$.

5) Calculate $\delta_{o}$ for each pixel in the object image.

This procedure is repeated for each backprojection image since $(1+f)$ and $\delta_{s}$ will change from image to image. 


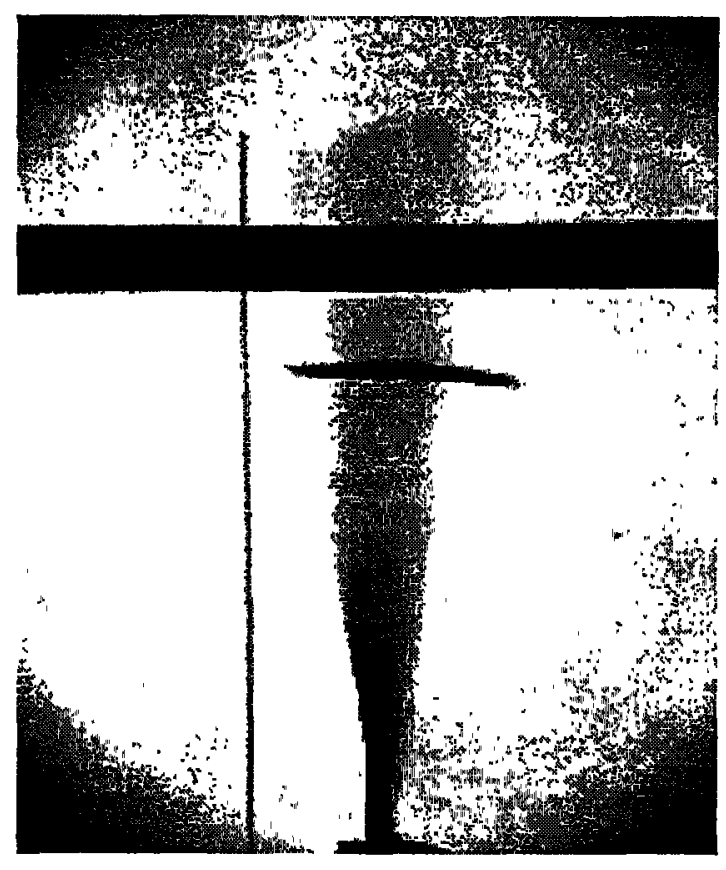

Figure 3.1: Typical image of a compressor blade before noise correction and normalization. The drop in beam intensity can be seen at the edges of the image. The borated polyethylene can be seen as the dark stripe across the image. The rod to the left of the blade is a centering rod. The rod is removed during backprojection image acquisition.

\subsection{Beam attenuation and hardening}

The tomographic reconstruction algorithm calculates the spatially dependent attenuation coefficient, $\mu(x, y, s)$, from the line integrals, $p(\theta, x, y)$, in the backprojection images[kak88].

$$
p(0, x, y)=\int_{-\infty}^{\infty} \mu(x, y, s) d s
$$

The coordinates $x$ and $y$ designate the position on the scintillator screen, $s$ is the path along the beam and perpendicular to the screen, and 0 is the angular rotation of the object. The line integrals are found from the attenuation of the 


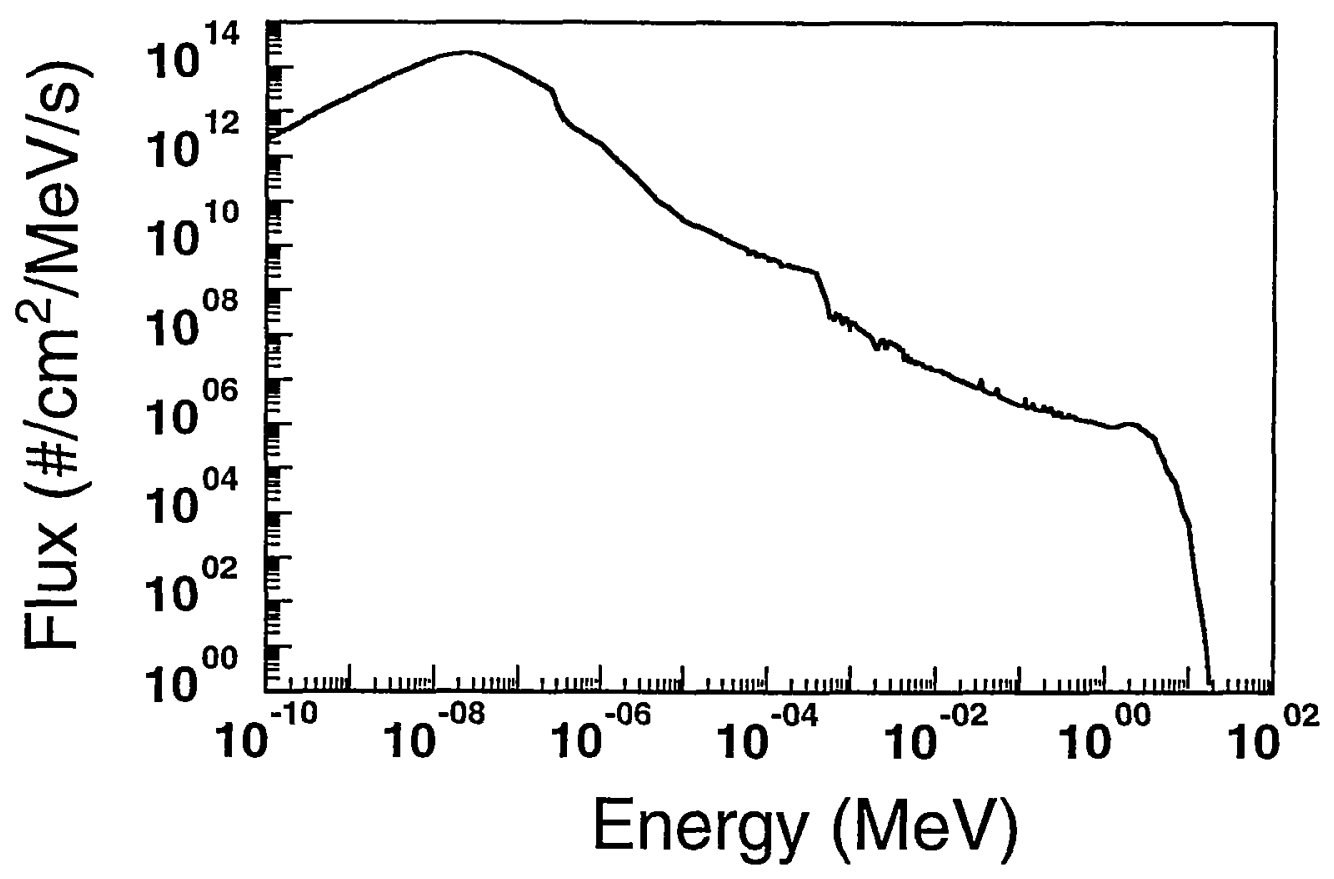

Figure 3.2: Neutron flux $\left(\frac{\|}{\pi} / \mathrm{cm}^{2} / \mathrm{MeV} / \mathrm{s}\right)$ energy spectrum in the neutron beam.

beam intensity as it passes through an object which for a monoenergetic beam is

$$
I_{o}(0, x, y)=I_{b}(0, x, y) \exp (-p(\theta, x, y))
$$

The density of the attenuating material can then be found from

$$
n(x, s)=\mu(x, s) / \sigma\left(E_{o}\right)
$$

where $\sigma\left(E_{o}\right)$ is the neutron cross section at energy $E_{o}$.

Unfortunately, the neutron beam provided by the MNRC reactor is not monoenergetic. The spectrum has a distribution similar to a Maxwellian in the thermal region, a distribution proportional to $1 / \mathrm{E}$ above $0.5 \mathrm{eV}$, and a bump in the fast region around $2 \mathrm{MeV}$. Above $10 \mathrm{MeV}$ the distribution drops to zero rapidly. The spectrum, as delermined through foil measurements[boo79][gilo55] and calculation with the code SANDII[mce67][kel87], is shown in figure 3.2. Because of the beam energy distribution, the energy dependence of the neutron cross 
sections for the sample and the scintillator screen becomes important. The general trend is for cross sections to decrease as the energy increases. This is useful in the detector since higher energy parts of the spectirum will not be absurbed. In the sample materials this trend leads to beam hardening. As the sample becomes thicker, the lower energy parts of the spectrum are depleted at a faster rate than the higher energy parts because of the larger cross section at low cnergy.

The effect of the hardening is shown in figure 3.3 for samples of titanium alloy Ti6 Al4V and polyethylene. Disks of titanium alloy were obtained from the National Institute of Standards and Technology (NIST). The $3.5 \mathrm{~cm}$ diameter disks were stacked end to end to provide different thicknesses for attenuation. A step wedge of polyethylene with a hydrogen density of $7.8 \cdot 10^{22} / \mathrm{cm}^{3}$ was also imaged. The percent of each element by weight in the polyethylene is $60.02 \%$ $\mathrm{C}, 31.95 \% \mathrm{O}, 8.00 \% \mathrm{H}$, and $0.014 \% \mathrm{~N}$ with a density of $1.19 \mathrm{~g} / \mathrm{cm}^{3}[1]$. While the molecular structure in this material is not the same as hydrogen in titanium, the data does give an estimate for hydrogen attenuation equivalent to $29000 \mathrm{ppm}$ weight in titanium. The wedge is $2.54 \mathrm{~cm}$ thick, and the distance from step to step is $0.3175 \mathrm{~cm}$. The images were preprocessed using the procedures to correct for noise. The intensity was averaged over a number of pixels behind each object, and the objects were large enough to minimize effects from edge unsharpness. The attenuation coefficients were calculated from the measured intensities, the thickness of the samples, and eq. (3.13). As can be seen in the figire the exponential attenuation assumption carses a change in the effective attenuation coefficient for the titanium alloy of $12 \%$ for a $3 \mathrm{~cm}$ change in thickness. The effect in the polyethylene is even greater.

The attenuated intensity for a beam with energy distribution $f(F)$ is

$$
\frac{I_{o}(\theta, x, y)}{I_{b}(0, x, y)}=\frac{\int f(E) \exp \left(-p_{n}(\theta, x, y) \sigma(E)\right)\left(1-\exp \left(-p_{d}(E)\right)\right) d E}{\int f(E)\left(1-\exp \left(-p_{d}(E)\right)\right) d E}
$$

Here $p_{n}$ is the line integral of the sample density, and $p_{d}$ is the line integral of the detector attenuation coefficient. Given a function for the beam energy spectrum, eq. (3.15) can be solved for $p_{n}$. Note that $\sigma(E)$ was removed from the line integral. This is possible if all constituents of the sample have the same energy dependence for their cross sections. This is not the case for titanium and hydrogen; however, we are using this formulation in investigations of the beam hardening correction. For many structural materials $(\mathrm{Ti}, \mathrm{V}, \mathrm{W}, \mathrm{Fe}$ ) the low energy cross section is proportional to $1 / \sqrt{E}$ [sch $\check{5}$ ] while hydrogen has a low energy cross section proportional to $1 / E[\operatorname{sac} 41][$ mel49][mes51]. The cross section for Al has no 

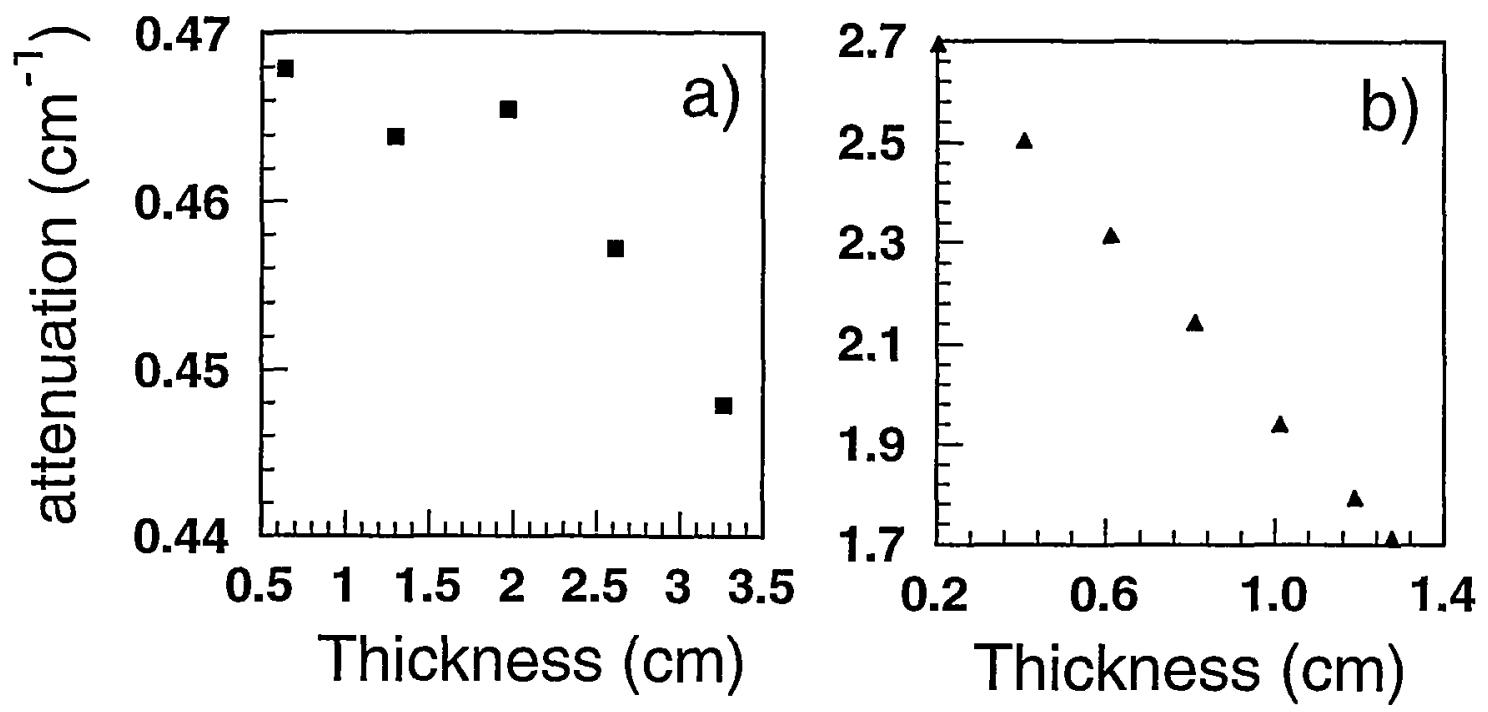

Figure 3.3: Attenuation coefficient $\left(\mathrm{cm}^{-1}\right)$ assuming exponential attenuation for a) Ti6 Al4V and b) polyethylene.

particular trend but several resonances at low energy[bro5̌8]. The general trend of increasing cross section with decreasing energy combined with low hydrogen concentrations should result in adequate correction of the beam hardening.

The correction for beam hardening in images is accomplished with a table of intensity versus titanium thickness. The table is plotted in figure 3.4 along with the exponential attenuation result, assuming an attenuation coefficient independent of energy. The difference between the two curves is not large for small thicknesses; however, the signals to be detected can cause changes in intensity as small as 0.005 . The table was generated by using eq. (3.15) to interpolate between the data points measured with the NIST titanium standards. Eq. (3.15) was fit to the data by changing the detector attenuation coefficient, and the ratio between the thermal and epithermal parts of the assumed beam energy spectrum. A precise match to the data from first principles was not attempted. Ecl. (3.15) was simply used as an interpolating function which was more physically consistent than a linear, splinc, or other interpolating function.

The preprocessing procedure for backprojection images is now complete. First 


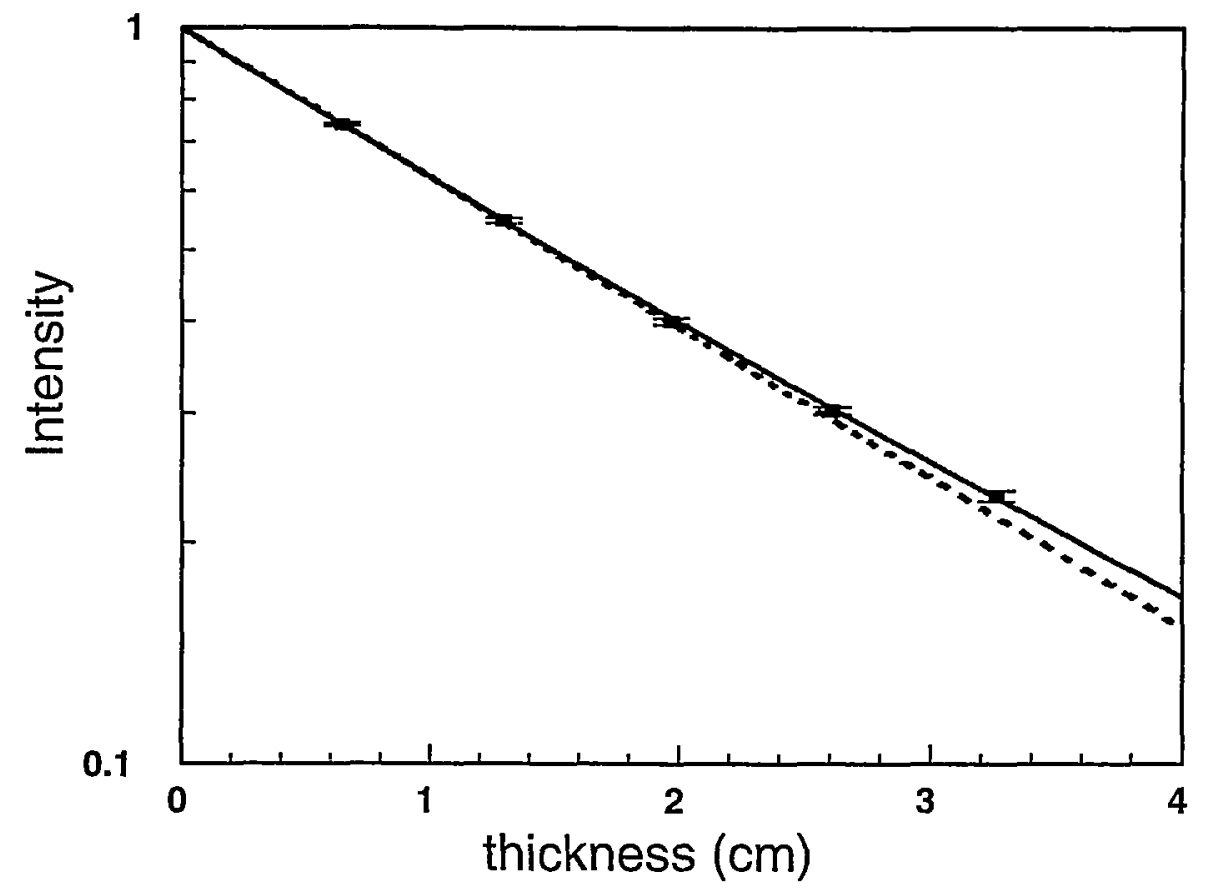

Figure 3.4: Dependence of intensity on Ti6Al4V thickness. The solid curve is the fit to the data points represented by squares. The dashed curve is the result assuming exponential attenuation and a constant attenuation coefficient.

a flat field image and a dark charge image are taken. The object to be reconstructed is then placed on the turntable, and the backprojection images are collected. The dark charge image is subtracted automatically during image acquisition. The images are preprocessed with the code PREPROC to correct for noise errors. Each image is divided by the flat field image. The code requires the user to supply the coordinates of square regions to be used for the beam intensity check and the scatter check. The background noise magnitude must also be provided. Smoothing of the image data can be applied according to the binomial 9-point filter $(4,2,1)$ [bir85]. The resulting images have intensities normalized to a maximum value of 1.0. The code then uses the attenuation table to convert the intensities in each pixel into equivalent thicknesses of the titanium alloy. The resulting pixel values are multiplied by 1000 . to provide convenient values to be 
copied to binary image files of 16 bits per pixel. These filcs are processed by the tomographic reconstruction program resulting in a plot of the material attenuation coefficient relative to Ti6Al4V without error due to beam hardening. Since we know the density of $\mathrm{Ti} 6 \mathrm{Al} 4 \mathrm{~V}$, the ratio of the alloy cross section to a sample material cross section gives the density of the sample material. The effective cross sections of materials must be found for our system by initially reconstructing samples of known material and density. The cross sections can then be used to determine samples of unknown density such as unknown concentrations of hydrogen in known metal components. For highly accurate measurements standard samples without hydrogen must be reconstructed first for comparison.

\subsection{The hydrogen signal}

Estimates are now needed for the hydrogen signal relative to the titanium alloy signal. The attenuation caused by a number of test objects is listed in table 3.1 under the intensity column. The first four objects are Ti6Al4V plates $(20 \times 10 \times$ $.343 \mathrm{~cm}^{3}$ ) loaded with hydrogen at Pratt and Whitney[pri94]. Plugs from each plate were tested with an inert gas fusion analyzer. In addition NIST performed Cold Neutron Prompt Gamma Activation Analysis (CNPGAA) on several spots of each plate[pau96]. The rest of the entries are from the polyethylene step redge. The values, ' $T_{e}$, in the effective thickness column result from the conversion of the measured intensities using the table of figure 3.4. The actual thicknesses, $T$, were measured with a micrometer. The Ti6 Al4V thickness is the thickness of each sample due to the titanium alloy. It is assumed that the hydrogen has not changed the thickness of the alloy plates. The effective thickness of the object due only to hydrogen, $d_{H}$, is found by subtracting $T$ from $T_{e}$.

The change in normalized density due to $100 \mathrm{ppm}$ hydrogen can be determined by

$$
\frac{n_{100 p p m I I}}{n_{T z}}=\frac{d_{H}}{T} \frac{100 p p m}{n_{H I}}
$$

Dividing by the actual hydrogen concentration in ppm, $n_{H}$, gives the normalized density per ppm. Multiplying by $100 \mathrm{ppm}$ scales the density convienently to the range of interest, for the embrittlement application. The normalized density, calculated for each sample, is in the last column of the table. According to the table, hydrogen at $100 \mathrm{ppm}$ will cause an increase in the normalized density of approximately $0.018 \div 0.002$. Subtracting the density in a standard without hydrogen from the density in a test sample and dividing by 0.018 will result in the 
hydrogen concentration in units of $100 \mathrm{ppm}$. The variation of $36 \%$ in the table 3.1 results indicates the need for further investigation to refine the magnitude of the hydrogen signal. 
Table 3.1: Hydrogen normalized density

$\begin{array}{ccccc}\text { Sample } & \text { Intensity } & \begin{array}{c}\text { Effective } \\ \text { Thickness } \\ (\mathrm{cm})\end{array} & \begin{array}{c}\text { Act.ual } \\ \text { Thickness } \\ (\mathrm{cm})\end{array} & \begin{array}{c}\text { Ti6Al4V } \\ \text { Thickness } \\ (\mathrm{cm})\end{array} \\ \text { Plate 115 } & 0.8557 & 0.3284 & 0.3276 & 0.3276 \\ \text { Plate 175 } & 0.8476 & 0.3486 & 0.3404 & 0.3404 \\ \text { Plate 4400 } & 0.5769 & 1.180 & 0.3353 & 0.3353 \\ \text { Plate 1735 } & 0.4583 & 1.689 & 0.3442 & 0.3442 \\ \text { Polyethylene } & 0.5785 & 1.174 & 0.2032 & 0.0 \\ \text { Polyethylene } & 0.3615 & 2.223 & 0.4064 & 0.0 \\ \text { Polyethylene } & 0.2438 & 3.130 & 0.6096 & 0.0 \\ \text { Polyethylene } & 0.1762 & 1.160 & 0.8103 & 0.0 \\ \text { Polyethylene } & 0.1407 & 4.920 & 1.011 & 0.0 \\ \text { Polyethylene } & 0.1187 & 5.510 & 1.189 & 0.0 \\ \text { Polyethylene } & 0.1122 & 5.710 & 1.277 & 0.0 \\ & & & & \\ \text { Sample } & \text { Hydrogen } & d_{H J} & \text { Normalized } & \\ & \text { concentration } & & \text { density } & \\ & (\mathrm{ppm}) & & & \\ \text { Plate 115 } & 115 \pm 30 & 0.0008 & 0.00212 & \\ \text { Plate 175 } & 170 \pm 20 & 0.0082 & 0.0142 & \\ \text { Plate 4400 } & 12900 \pm 900 & 0.8442 & 0.0195 & \\ \text { Plate 1735 } & 17900 \pm 700 & 1.345 & 0.0218 & \\ \text { Polyethylene } & 29000 & 1.174 & 0.0199 & \\ \text { Polyethylene } & 29000 & 2.223 & 0.0189 & \\ \text { Polyethylene } & 29000 & 3.130 & 0.0177 & \\ \text { Polyethylene } & 29000 & 4.160 & 0.0177 & \\ \text { Polyethylene } & 29000 & 4.920 & 0.0168 & \\ \text { Polyethylene } & 29000 & 5.510 & 0.0160 & \\ \text { Polyethylene } & 29000 & 5.710 & 0.0154 & \\ & & & & \end{array}$




\section{TOMOgRAPHIC RECONSTRUCTION RESULTS}

Reconstruction was accomplished with the filtered backprojection routine from the Donner algorithms library[hue77]. The exposure time for each backprojection image was 6.2 seconds. Another 13 seconds was required for downloading and dark charge subtraction by the PC. The reconstructions shown below were calculated from sets of 180 backprojections ( $1^{\circ}$ increments) resulting in an image acquisition time of 60 minutes. As mentioned above the code PREPROC performs the preprocessing procedures on the images. The code IMIGPROC does the 2-D reconstruction from desired slices of the original images. This section contains results from the reconstructions of a NIST titanium standard, a phantom with various metals, and an engine compressor blade. The reconstructions quantify the reported magnitude of the titanium alloy density as well as fluctuations due to noise. The response to metals with different attenuation coefficients indicates the ability to evaluate components made of various materials. The blade reconstruction shows the result for thin objects.

\subsection{NIST titanium alloy standard}

One of the titanium alloy standards, previously used in the measurements of attenuation coefficient, was reconstrucled as shown in figure $4.1 \mathrm{a}$ and $\mathrm{b}$. The standard is a circular disk $3.5 \mathrm{~cm}$ in diameter and $1.97 \mathrm{~cm}$ thick. The axis of the disk was placed perpendicular to the rotation axis resulting in a rectangular cross section in the reconstruction. The color scale was set in the figure to only include densities from the maximum to $15 \%$ below the maximum which heightens the contrast for the features inside the standard. Figure 4.1a is the result without image corrections and attenuation calculated using the exponential approximation. Figure $4.1 \mathrm{~b}$ is the result with all of the corrections. The first reconstruction has attenuation coefficient in units of $\mathrm{cm}^{-1}$ while the second reconstruction has 

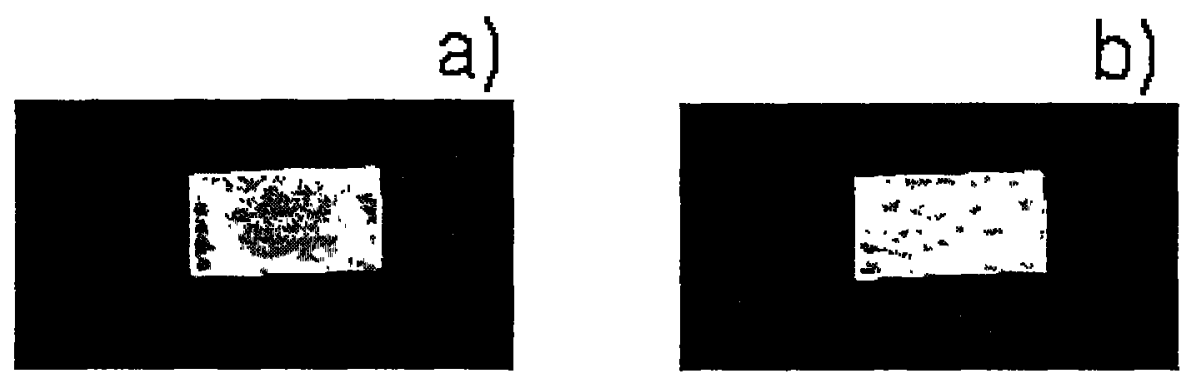

Figure 4.1: Reconstruction of the NIST Ti6Al4V standard with a) no corrections and b) all corrections

attenuation coefficient normalized to the titanium alloy.

The main feature of the uncorrected reconstruction is the larger attennation coefficient around the edges versus the center. This result is typical in cases where beam hardening is significant[kak88][mcd77]. The backprojection images show a relatively higher attenuation coefficient for those line integrals which pass through less material. The tomography algorithm responds to this by partially "hollowing out" what should be a uniform density object. Both the actual beam hardening and the background signals contribute to this effect. Figure $4.2 \mathrm{a}$ is a curve of the density across the center of the disk showing the magnitude of the effect. The edges of the disk have an attenuation coefficient approximately $10 \%$ larger than the center, a change from $0.37 \mathrm{~cm}^{-1}$ to $0.41 \mathrm{~cm}^{-1}$. If we did not know that this NIST standard has less than $50 \mathrm{ppm}$ hydrogen in it, we might conclude that up to $500 \mathrm{ppm}$ hydrogen had diffused into the surface layers of the disk. The elimination of the beam hardening artifacts is obvious in the corrected image reconstruction. The normalized density curve in figure $4.2 \mathrm{~b}$ shows a flat profile across the standard. The average density is 1.008 , and the noise has a standard deviation of \pm 0.008 or $\pm 50 \mathrm{ppm}$.

\subsection{Cylindrical phantom}

The next set of experiments involved a phantom constructed from an aluminum cylinder $4 \mathrm{~cm}$ in diameter and $10 \mathrm{~cm}$ long. It is similar to experiments reported 

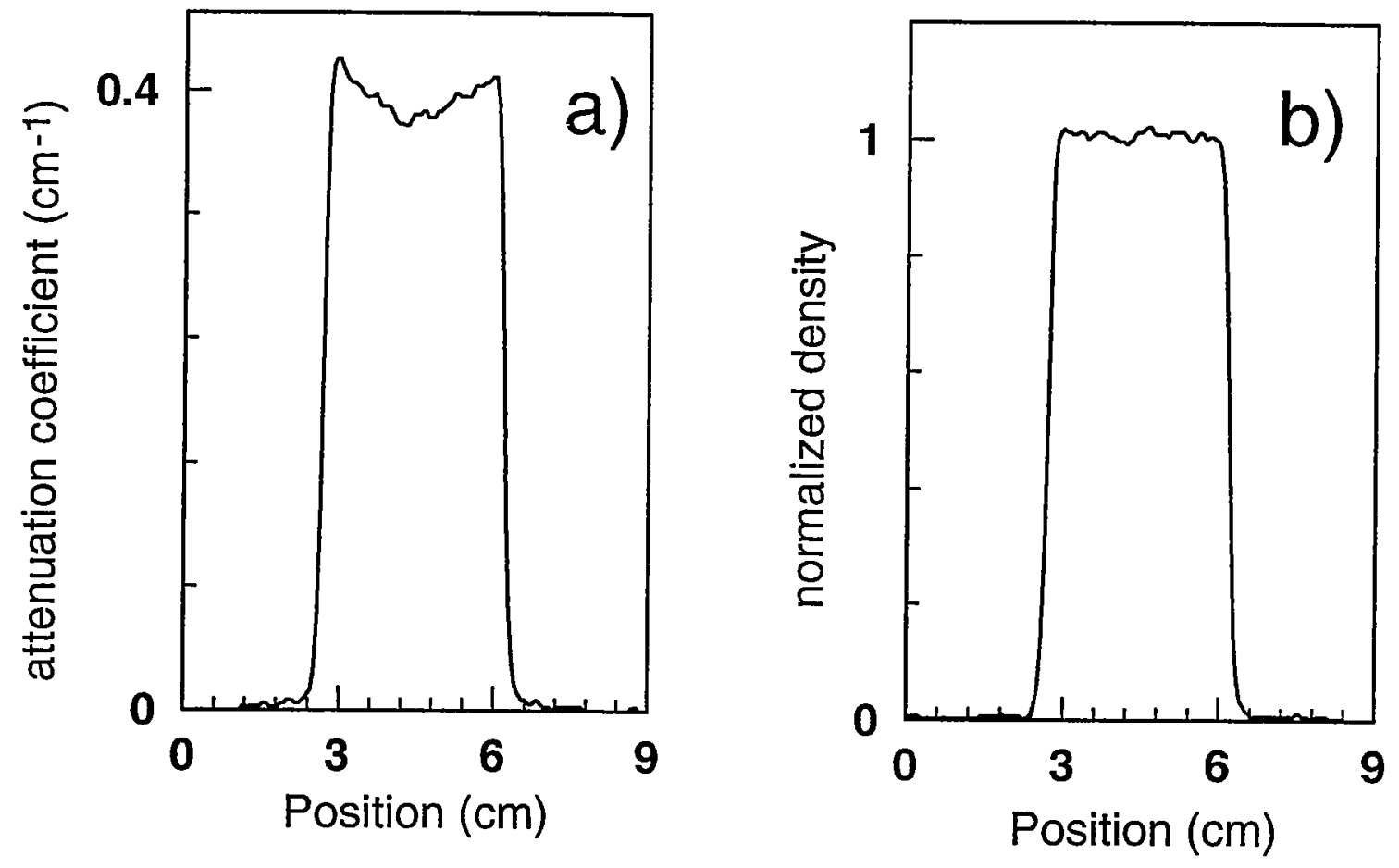

Figure 4.2: Attenuation coefficient across the center of the disk for the a) uncorrected and b) corrected backprojection images. 
by Zanarini, et. al.[zan95]; however; the differences in configurations highlight; some of the features of the MNRC tomography system. Their experiments used a cold neutron beam which eliminates the energy corrections necessary in the MNRC system. This also results in a smaller, $4 \mathrm{~cm}$ diameter, and lower flux, $8 \cdot 10^{5} \mathrm{n} / \mathrm{cm} 2 \mathrm{~s}$, beam. This beam would not be sufficient to acquire images for the compressor blades in a production mode. The large MNRC beam has deficiencies, background noise and hardening, which can be corrected to provide a production capable system.

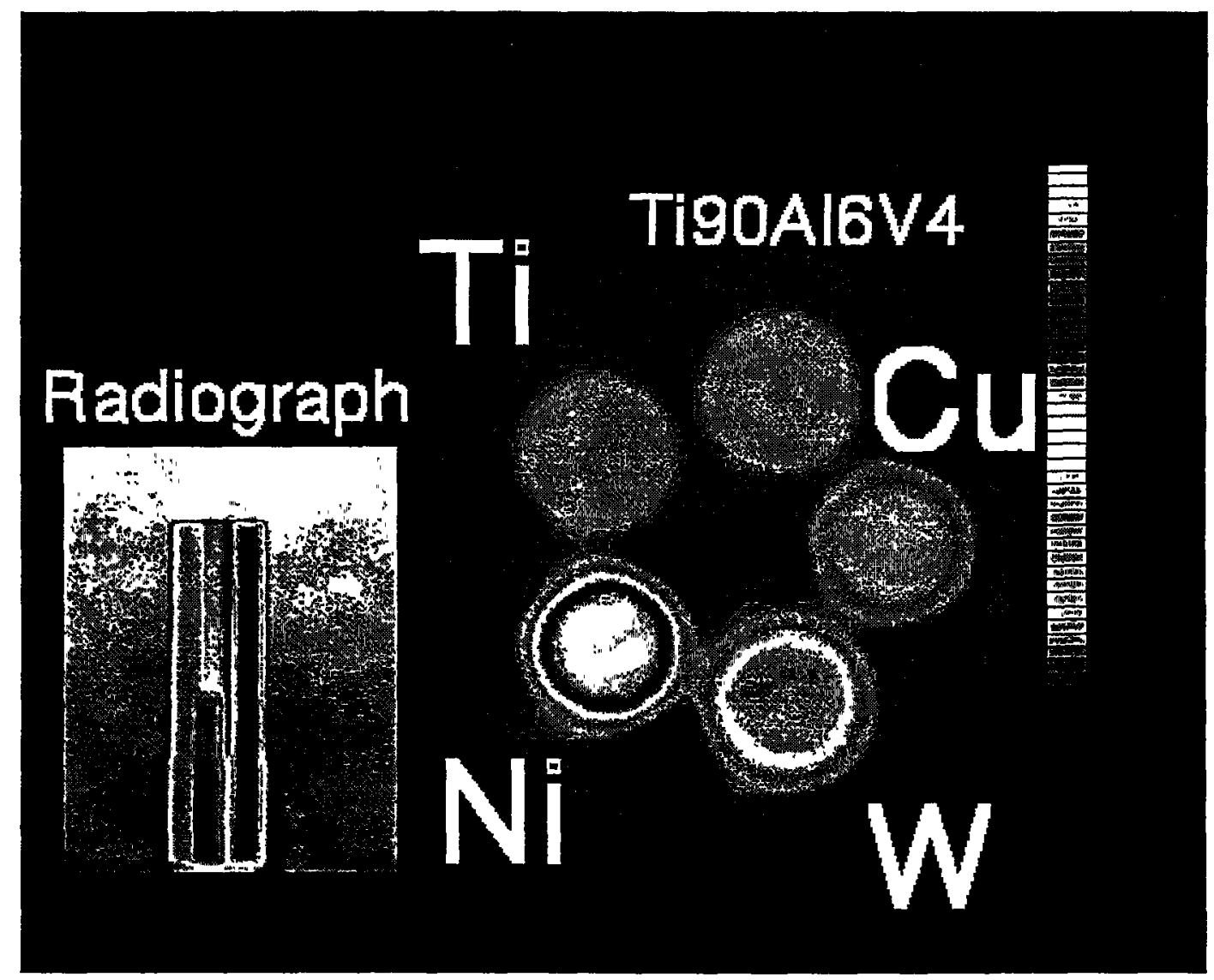

Figure 4.3: Reconstruction of aluminum cylinder with Ti90Al6V4, $\mathrm{Ti}, \mathrm{Cu}, \mathrm{W}$, and $\mathrm{Ni}$ rods. A backprojection image is also shown.

The aluminum phantom had five holes with diameters of $1 \mathrm{~cm}$. The first 
Table 4.1: Relative attenuation coefficients for different metals

$\begin{array}{ccc}\text { Sample } & \begin{array}{c}\text { Measured } \\ \text { Coefficient, }\end{array} & \begin{array}{c}\text { Calculated } \\ \text { Coefficien } \\ \left(\mathrm{cm}^{-1}\right)\end{array} \\ \text { Ti90Al6V4 } & 1.022 \pm 0.018 & 0.044 \\ \mathrm{Ti} & 1.089 \pm 0.013 & 0.573 \\ \mathrm{Cu} & 1.883 \pm 0.017 & 0.993 \\ \mathrm{~W} & 2.486 \pm 0.062 & 1.163 \\ \mathrm{Ni} & 4.270 \pm 0.064 & 1.984\end{array}$

phantom reconstruction in figure 4.3 has rods of $\mathrm{Ti} 6 \mathrm{Al} 4 \mathrm{~V}, \mathrm{Ti}, \mathrm{Ni}, \mathrm{W}$, and $\mathrm{C} n$ in the holes. This serves as a check of the preprocessing algorithm for materials with attenuation coefficients different than titanium. The transition in color from bluegreen to pink represents a change in effective attenuation coefficient by a factor of four. Even with this large change in attenuation coefficient the reconstruction results in a fairly uniform density across each rod. The variation is less than $2 \%$ of the average density for each rod. Since the absolute magnitude of the noise increases for the higher attenuation coefficient materials, the minimum detectable hydrogen concentration will also increase for these materials.

As a check for consistency, the attenuation coefficients $\left(\mathrm{cm}^{-1}\right)$, based on the thermal neutron cross sections and material densities, are compared to the measured attenuation coefficients. The data is listed in table 4.1 and plotted in figure 4.4. The close fit to a linear relationship indicates that the attenuation coefficients are being reconstructed correctly even for materials causing significant attenuation of the beam. The beam intensity is 0.63 after atlenuation by $1.0 \mathrm{~cm}$ of $\mathrm{Ti}$ and 0.17 after attenuation by $1.0 \mathrm{~cm}$ of $\mathrm{Ni}$. These results are similar to Ikeda, et. al.[ike90]. Again the measured attenuation coefficients and calculated cross sections are not the same since the measured values are normalized to the titanium alloy during image preprocessing. The most important result is the uniformity of the reconstructed density across each of the rods. A wide range of components can be evaluated with the system in its present configuration. 


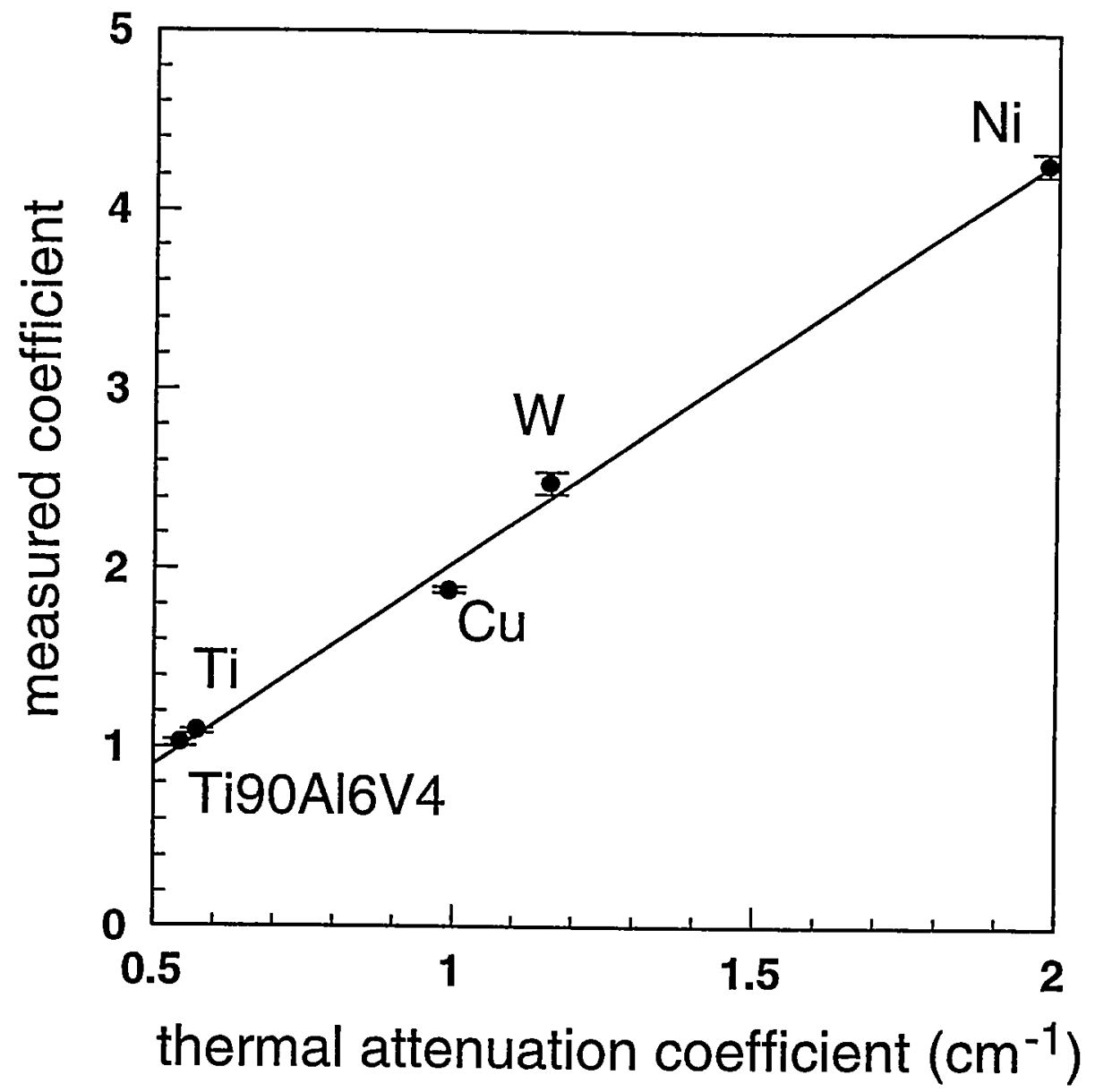

Figure 4.4: The measured attenuation coefficient, normalized to the titanium alloy during image preprocessing, plotted versus the thermal neutron attenuation coefficient for several metals. 


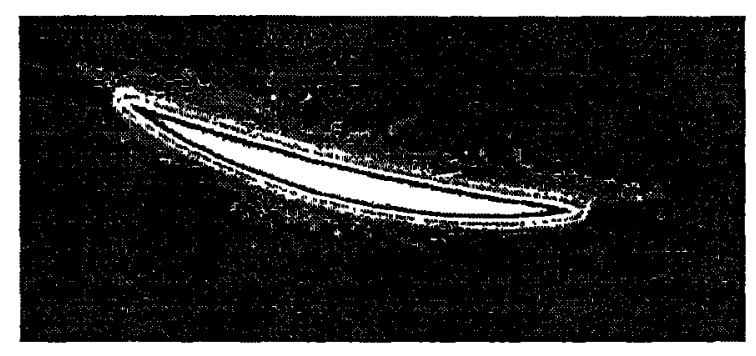

Figure 4.5: Tomographic reconstruction of a compressor blade.

\subsection{Compressor blade reconstruction}

The result of the reconstruction for a new engine compressor blade is shown in figure 4.5. In this picture the color scale includes all attenuation coefficient values so the reconstruction artifacts can be seen. Notice that the radial variations in the blade correspond to the radial artifacts in the reconstruction. These radial artifacts are due to the finite number of backprojection images. The interior region of the blade does not suffer too badly from these artifacts. The density is $1.009 \pm 0.008$ which represents a variation of only $\pm 50 \mathrm{ppm}$ hydrogen. This signal is so low that ne cannot be certain if it is due solely to noise or if there is some hydrogen signal present. Since the requirement is to detect levels only down to 100 to $200 \mathrm{ppm}$, the level of noise appears tolerable. At least as far as the interior of the blade is concerned, the shape of the blade has not adversely impactied the hydrogen detection capability. This was not the case for reconstructions generated from 60 backprojection images. In the 60 image case the radial artifacts caused variations equiralent to a signal due to $400 \mathrm{ppm}$ hydrugen. Because of these artifacts the choice was made to use 180 images at the cost of a longer acquisition time.

Cross sections of the blade reconstruction are in figure 4.6a and b. Again in the interior of the blade, the effective density is near 1.0 as required for the titanium alloy. These curves illustrate the limits of spatial resolution in the reconstructions. Curve a) passes across the thin dimension of the blade while curve b) passes along the width of the blade. In curve a) the blade is only 10 to 12 pixels thick and the edges are seen to spread over 4 pixels. The dashed line represents a hypothetical reconstruction with no edge spread. The result is the degradation of the density signal for two pixels or $\sim 1 \mathrm{~mm}$ inward from the edge of the object. Since the blade 

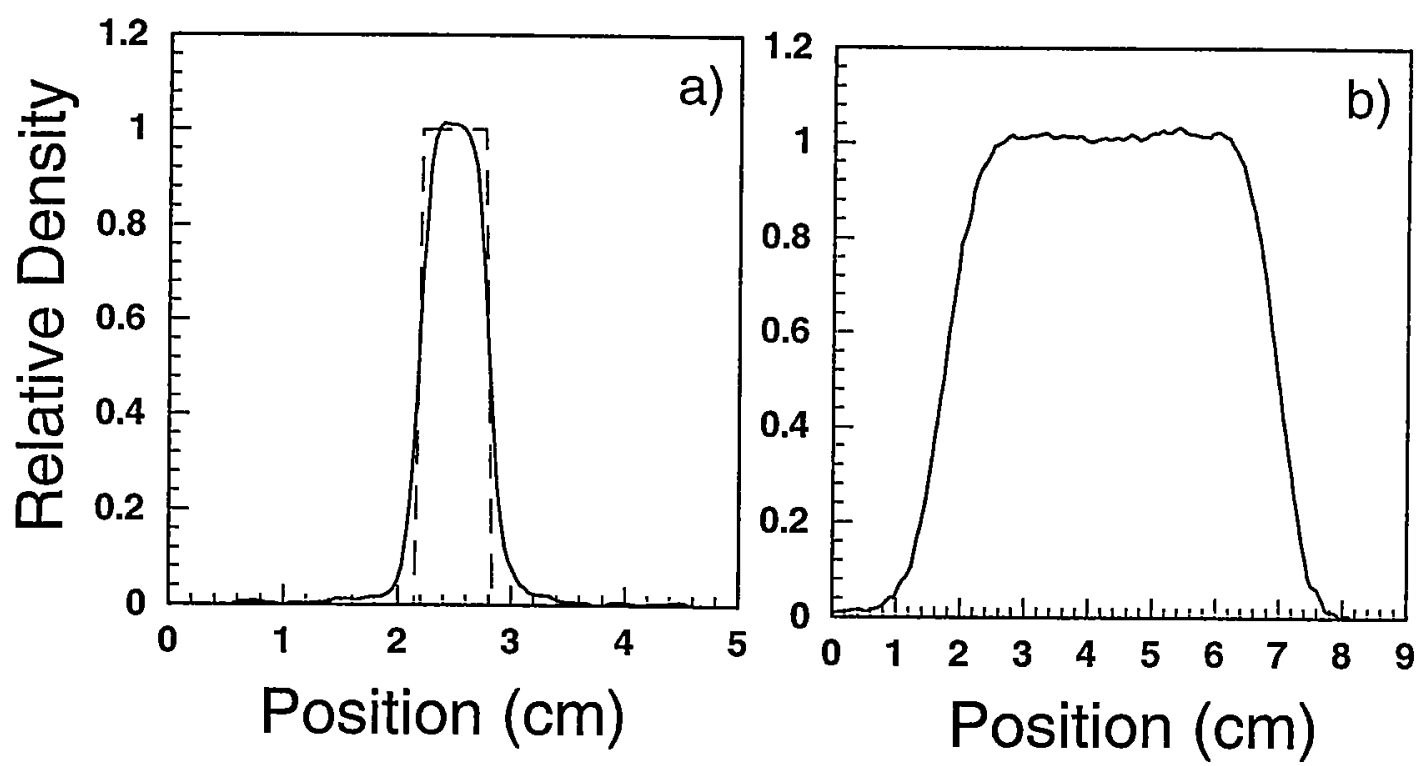

Figure 4.6: Cross section of engine compressor blade reconstruction a) across the thickness of the blade and $b$ ) across the width of the blade.

has a crescent shape, the width of the edges are exaggerated in curve b). The spatial resolution limits could be a problem if hydrogen diffuses only into a thin layer on the surface of parts. If the hydrogen mobility is sufficient to diffuse more evenly through a part, the spatial resolution should be adequate. 


\section{Conclusion}

The MNRC neutron tomography system has demonstrated the capability to detect hydrogen in titanium components to levels of $100 \mathrm{ppm}$ weight with an acquisition time of 60 minutes. Since the turntable fixtures accomodate three engine compressor blades, nondestructive testing can be accomplished at a rate of three blades per hour. These testing characteristics were achieved by using a large, thermal beam with preprocessing of backprojection images to correct for image errors. The preprocessing included correction for CCD camera dark charge, correction for background, correction for reactor fluctuations, correction for scattering, and correction for beam hardening.

Further work has been completed to address the remaining artifacts in reconstruction images. Comparison of standards without hydrogen to objects with unknown hydrogen will be used to detect hydrogen signals even in parts of the reconstruction which hare artifacts. The standard and test objects will have the same physical dimensions and metal matrix. The difference between the two reconstruction images should reveal the hydrogen signal assuming the artifacts remain the same. The artifacts should remain essentially unchanged given small hydrogen signals. Large hydrogen signals may have a significant impact on the artifacts, but the large signals will be easy to detect above the noise. The techniques for reconstruction image comparison will be addressed in a future report. Standards with various levels of hydrogen are also being developed so the magnitude of the hydrogen signal can be quantified more precisely. 


\section{ACKNOWLEDGMENTS}

This work would not hare been possible without the initial request by the safety office of HQ AFMC for blade inspection, and the decision to take up the challenge by Wade J. Richards, chief of the MNRC. Jim Crump and the rest of the staff at the MINRC were responsible for the construction and the initial work done with the tomography system. SMI-ALC/TIE provided an interface to the LBL tomography routines. J.P. Barton provided useful discussions, and E.W. McFarland tested an initial CT system in radiography bay 4. We also thank R.L. Paul, M. Privett, R.M. Lindstrom, and MI. Arif for the titanium plate results. This work was jointly supported by the U.S. Air Force and by the U.S. Department of Energy. Lawrence Livermore National Laboratory under contract number W-7405-ENG-48. 


\section{BIBLIOGRAPHY}

[alv90] K. Alvar, T. Maung, V. Orphan, and R. Polichar, "Neutron Radiography with SNRS," Neutron Radiography (3), Kluwer Academic Publishers, 439$446,1990$.

[bir85] C.K. Birdsall and A.B. Langdon, "Plasma Physics vis Computer Simulation," McGraw-Fill Book Co., New York, NY, 437-441, 1985.

[boo79] S.J. Boot, "Calibration of Reference Thermal and Epithermal Neutron Flux Densities from the GLEEP Reactor at AERE Harwell," Nucl. Inst. and Meth. 164, 513-519 (1979).

[bro58] R.J. Brown and L.M. Bollinger, "Total Neutron Cross Sections for Structural Materials," Nucl. Sci. Eng. 4, 576-580 (1958).

[1] B. Connolly, "Composition of Polycast clear PMIMA shects," letter from Polycast Technologies, 70 Carlisle Place, Stamford, CT 06902, Sept. 28, 1995 .

[gib95] M.R. Gibbons and N.L. Newell, "Neutron Flux Energy Spectra in the MINRC TRIGA Reactor Radiography Bays," McClellan Nuclear Radiation Center report MNRC-0057-DOC-00, Sep. 1995.

[fro90] D.A. Froom and J.P. Barton, "Aircraft Neutron Radiography-an Overview," Neutron Radiography (3), Kluwer Academic Publishers, 293:t, 1990 and other articles in Part 13: Aerospace Applications

[har86] A.A. Harms and D.R. Wyman, "Mathematics and Physics of Neutron Radiography," D. Reidel Publishing Co., Dordrecht, Holland, 1986.

[hue77] R.H. Heusman, G.T. Gullberg, W.L. Greenberg, and T.F. Budinger, "Donner Algorithms for Reconstruction Tomography," Lawrence Berkeley Laboratory, University of California, Pub. 214, October 1977. 
[ike90] Y. Ikeda, A. Ando, K. Ohkubo, M. Yokoi, and H. Kobayashi, "A New Imaging Device for Neutron CT, "Neutron Radiography (3), Kluwer Academic Publishers, Dordrecht, Holland, 835-842, 1990.

[ike96] Y. Ikeda, M. Yokoi, M. Oda, M. Tamaki, and G. Matsumoto, "Correction of Scattering Neutron effects on Neutron CT," Nucl. Inst. and Meth. A 377, 85-89 (1996).

[kak88] A.C. Kak and M. Slaney, Principles of Computerized Tomographic Imaging, IEEE Press, New York, NY, 1988.

[kel87] J.G. Kelly and D.W. Vehar, "Measurement of Neutron Spectra in Varied Environments by the Foil-Activation Method with Arbitrary Trials," Sandia National Laboratory report SAND87-1330, Dec. 1987.

[mcd77] W.D. McDavid, R.G. Waggener, W.H. Payne, and M.J. Dennis, "Correction for Spectral Artifacts in Cross-sectional reconstruction from X-rays," Med Phys. 4, 54-57 (1977).

[mce67] M.N. McElroy, S. Berg, T. Crockett, and R.G. Hawkins, "A Computer Automated Iterative Method for Neutron Flux Specta Determination by Foul Activation," AFWL-TR-67-41, Atomics International, Sep. 1967.

[mcf91] E.W. McFarland, R.C. Lanza, and G.W. Poulos, "Multidimensional Neutron-Computed Tomography using Cooled Charge-coupled Devices," IEEE Trans. on Nucl. Sci. 38, 612-622 (1991).

[mel49] E. Melkonian, "Slow Neutron Velocity Spectrometer Studies of $\mathrm{O}_{2}, \mathrm{~N}_{2}$, A, $\mathrm{H}_{2}, \mathrm{H}_{2} 0$, and Seven Hydrocarbons," Phys. Rev. 76, 1750-1759 (1949).

[mes51] A.M.L. Messiah, "Scattering of Slow Neutrons by $\mathrm{H}_{2}$ and $\mathrm{CH}_{4}$," Phys. Rev. 84, 204-214 (1951).

[nel83] H.G. Nelson, "Hydrogen Embrittlement," Treatise on Materials Science and Technology, vol. 25, Academic Press, New York, NY, 275-359,1983.

[net94] private communication, NE Technology, Inc., Monmouth Junction, NJ 08852

[nus68] A. Nussbaum, "Geometric Optics: An Introduction," Reading, MA, Addison-Wesley Publishing Co., 72-87, 1968. 
[pat74] N.E. Patton and J.C. Williams, "Effects of Hydrogen on Titanium and its Alloys," Hydrogen in Metals, Metals Park, OH, American Society for Metals, 1974, 409-432.

[pim93] "TE/CCD Detector Operation Manual" and "ST-138 Controller Operation Manual," Pinceton Instruments, Inc., Trenton, NJ, 1993.

[pre88] W.H. Press, B.P. Flannery, S.A. Teukolsky, and W.T. Vetterling, "Numerical Recipies," Cambridge Úniversity Press, 1988.

[pri94] M. Privett, "titanium/hydrogen panel standards," letter from Lnited Technologies Pratt\&Whitney, P.O. Box 109600, West Palm Beach, FL 33410-9600, June 17, 1994.

[pau96] R.L. Paul, MI. Privett, R.M. Lindstrom, and W.J. Richards, and R.R. Greenberg; "Analysis of Hydrogen in Titanium Alloys by Cold Neutron Prompt Gamma Activation Analysis," Metall. Mater. Trans. A, 27A, 3682-3687 (1996).

[ric90] W.J. Richards, "Stationary Neutron Radiography System," Trans. Am. Nucl. Soc., 62, 142-143 (1990).

[sac41] R.G. Sachs and E. Teller; "The Scattering of Slow Neutrons by Molecular Gases," Phys. Rev. 60, 18-27 (1941).

[sch59] R.E. Schmunk, P.D. Randolph, and R.M. Brugger, "Total Cross Sections of Ti, V, Y, Ta, and W," Nucl. Sci. Eng. 7, 193-197 (1960).

[spo69] A.R. Spowart, "Measurement of the Absolute Scintillation Efficiency of Granular and Glass Neutron Scintillators," Nucl. Insl. and Meth. A 75, $3 \tilde{\jmath}-42(1969)$.

[zan95] M. Zanarini, P. Chirco, M. Rossi, G. Baldazzi, G. Guidi, E. Querzola, M.G. Scannavini, F. Casali, A. Garagnani, and A. Festinesi, "Evaluation of Hydrogen content in Metallic Samples by Neutron Computed Tomography," IEEE 'Trans. Nucl. Sci. 42, 580-584 (1995). 